\title{
MODELOS DE ORGANIZACIÓN POLÍTICA EN CRETA NEOPALACIAL: UNA APROXIMACIÓN INTERCULTURAL DESDE LA ARQUEOLOGÍA MAYA
}

\section{MODELS OF POLITICAL ORGANIZATION IN NEOPALATIAL CRETE: A CROSS-CULTURAL APPROACH FROM MAYAN ARCHAEOLOGY}

\author{
Jorge CANO MORENO
}

\author{
Consejo Nacional de Investigaciones Científicas y Técnicas (CONICET), Universidad Católica Argentina \\ (UCA), Ciudad Autónoma de Buenos Aires \\ Correo electrónico: canomorenojj@gmail.com
}

\begin{abstract}
Resumen: En este trabajo analizaremos hasta qué punto la arqueología de la cultura maya puede ser útil para esclarecer el debate acerca de la organización e interacción política en la isla de Creta durante el período Neopalacial. Esta analogía no es caprichosa; dado que este tópico ha generado intensos debates en el campo de la arqueología cretense, algunos investigadores han considerado que los modelos empleados para las tierras bajas de Mesoamérica pueden ser de utilidad para brindar nuevas respuestas. Sin embargo, consideramos que no se ha profundizado lo suficiente en este tipo de comparación. Ante esta situación, nuestra investigación tiene cuatro objetivos generales: primero, ofrecer algunas bases teórico-metodológicas para realizar este análisis analógico; segundo, describir brevemente el estado de la cuestión existente en los estudios minoicos sobre el debate de la organización política; tercero, sistematizar los diferentes modelos propuestos para la cultura maya considerando sus bases epistemológicas y hermenéuticas; y, por último, hacer una evaluación acerca de los méritos (y defectos) que tiene el empleo de esta analogía.
\end{abstract}

Palabras Clave: Arqueología minoica, Arqueología maya, modelos políticos, interdisciplinariedad, cross-cultural Comparison.

\begin{abstract}
In this work, it will be analyzed to what extent the archaeology of the Mayan culture can be useful to clarify the debate about the organization and political interaction on the island of Crete during the Neopalatial period. This analogy is not whimsical; since this topic has generated intense debates in the field of Cretan archaeology, some researchers have considered that the models used for the lowlands of Mesoamerica may be useful to provide new answers. However, this type of comparison has not been studied in sufficient depth. Faced with this situation, this research has four general objectives: first, to offer some theoretical-methodological bases to carry out this analogical analysis; second, briefly describe the state of the art in Minoan studies on the debate about the political organization; third, to systematize the different models proposed for the Mayan culture considering their epistemological and hermeneutical bases; and, finally, evaluate the merits (and defects) of using this analogy.
\end{abstract}

Keywords: Minoan Archaeology, Mayan Archaeology, political Models, interdisciplinarity, cross-cultural Comparison.

Sumario: 1. Introducción. 2. Una breve aclaración metodológica. 3. Modelos políticos para Creta minoica. 3.1. El modelo de Evans. 3.2. El modelo de Peer Polities Interaction. 3.3. Nuevas críticas y nuevos modelos. 3.4. Facciones y competencia. 3.5. Antropología y households. 4. Debates sobre la organización política maya. 4.1. Las primeras síntesis. 4.2. Los modelos descentralizados. 4.3. Las críticas al estado segmentario y la configuración del modelo centralizado. 4.4. Entre la descentralización y la centralización: los modelos alternativos. 4.5. Enseñanzas de la arqueología Maya. 5. Conclusiones: Mayas, Minoicos y modelos políticos. 6. Agradecimientos. 7. Bibliografía. 


\section{Introducción}

Uno de los temas que más debates ocasiona en el campo de la arqueología de Creta durante la Edad de Bronce (también conocido como período minoico [Karadimas y Momigliano, 2004]) es el análisis de la organización política de la isla en el período Neopalacial (ca. 1700-1450 a.C.). Muchos de los puntos en cuestionamiento se originaron por las falencias que presentaba el esquema ideado por Arthur Evans en The Palace of Minos at Knossos publicado entre 1921-1938, en el cual se ubicaba al "palacio" de Cnosos como el centro del poder insular y como la sede de un imperio marítimo (talasocracia) que se extendía por la cuenca del mar Egeo según la tradición transmitida por algunos escritores clásicos.

Esta última afirmación fue la primera en ser refutada: algunos arqueólogos (Kantor, 1947; Wace y Blegen, 1939) no encontraban materiales suficientes que prueben dicho dominio y la mayoría de los filólogos (Buck, 1962; Dow, 1967; Starr, 1955) dudaba del uso literal que el pionero inglés había hecho de las fuentes escritas (Caldesi, 2009; Cano, 2020). A partir de los ochenta, de la mano de las nuevas corrientes arqueológicas, se matizaron o descartaron muchas de las tesis de Evans respecto a un supuesto dominio minoico sobre algunos territorios de Mediterráneo Oriental dando lugar a enfoques más amplios y menos dogmáticos (Branigan, 1981; Davis, 2008; Hägg y Marinatos, 1984; Knappet y Nikolakopoulou, 2008; Melas, 1991; Niemeier, 1991).

Sin embargo, la cuestión de la organización política dentro de la isla de Creta ha tenido una suerte diferente ya que no hay ningún modelo que tenga el consenso suficiente para significar una verdadera alternativa al que presentara Evans. Respecto a este punto, uno de los objetivos de este trabajo es el de realizar un breve estado de la cuestión acerca de las propuestas existentes hoy en día.

En este sentido, consideramos que hay cuatro enfoques a tener en cuenta: el primero es el basado en las ideas de Evans con algunas modificaciones realizadas por los seguidores de sus ideas; el segundo surge a partir del libro publicado por Renfrew y Cherry (1986) Peer Polity Interaction and Sociopolitical Change, en el cual se aplica el modelo de interacción entre unidades políticas similares; el tercero de los enfoques, se basa en las ideas de Hamilakis (2002), quien utiliza a las facciones como base para las dinámicas de interac- ción política; y el último modelo, es el empleado por Driessen $(2010,2018)$ quien utiliza el concepto de "casa" -en inglés, household o también house society- como la lógica de interacción predominante entre los grupos de elite y las diferentes regiones de la isla.

Estos dos últimos modelos han significado un acercamiento a las ciencias antropológicas, pero consideramos que la arqueología maya se encuentra más desarrollada en el uso de perspectivas interdisciplinarias y por lo tanto es atractivo hacer la comparación entre ambos campos. Esto se debe a las posibilidades epistemológicas que ofrece el área maya: a la evidencia material arqueológica se le suma la posibilidad de interpretar la gran mayoría de las fuentes epigráficas, la disponibilidad de la documentación escrita posteriormente a la conquista española (códices, crónicas, leyes, relatos, etc.) y, particularmente, la existencia de comunidades mayas contemporáneas. La etnología, la etnohistoria y la arqueología hacen que diversos marcos teóricos antropológicos sean utilizados recurrentemente.

Nuestro siguiente objetivo es el de presentar los distintos modelos de organización e interacción política propuestos para la cultura maya haciendo un especial énfasis en el período clásico ( $c a$. 250-900 d.C.), pero haciendo referencia a otros momentos de la historia como el período posclásico (ca. 950-1539 d.C.). Al no ser nuestra área de especialización, no nos detendremos a estudiar su pertinencia interpretativa respecto a la evidencia material, sino que más bien, nos preocuparemos en analizar sus bases epistemológicas y las posibilidades hermenéuticas que puedan sernos útiles.

El último objetivo del trabajo es evaluar qué aspectos de los estudios mesoamericanos pueden llegar a ser fructíferos a la hora de analizar la arqueología minoica. Es importante aclarar que no es nuestra intención realizar una comparación visa-vis entre ambos campos de estudios como si la cultura maya y la minoica (y sus respectivos períodos históricos) fueran unidades étnicas definidas y cronológicamente delimitadas. Catalogar un conjunto social y una etapa temporal con un epíteto determinado puede llegar a ser un ejercicio riesgoso por dos razones principales: por un lado, dichas denominaciones suelen sernos heredadas a través de tradiciones académicas que, más allá de sus méritos, pueden incluir falencias implícitas en los paradigmas que las sostienen. Además, este tipo de clasificaciones tienden a enfatizar las 
similitudes y a matizar las diferencias del registro arqueológico; en post de cierta coherencia unificadora, muchas veces, se han generado visiones monolíticas sobre distintos grupos humanos que han entorpecido la comprensión sobre sus vicisitudes históricas (Adams, 2004, 2006).

De todos modos, somos conscientes que es muy difícil -o más bien, imposible- estudiar a determinado grupo cultural sin caer en algún tipo de generalización ya sea desde la nomenclatura cultural, los enfoques teóricos o el análisis de la evidencia material (Politis, 2002). No obstante, consideramos que lo importante es tener la "vigilancia epistemológica" que propone Bourdieu (Bourdieu et al., 2004): reflexionar críticamente sobre los enfoques teóricos utilizados en determinado campo de estudio y sobre la propia posición del investigador respecto al objeto que estudia y sobre sí mismo en la sociedad. Con esta problemático comenzamos el siguiente apartado con la intención de sentar las bases que nos permitan realizar una correcta analogía.

\section{Una breve aclaración metodológica}

El primer objetivo de este trabajo es establecer las bases metodológicas que avale la posibilidad de realizar un análisis comparativo y analógico entre estos grupos humanos, lo que en el ámbito angloparlante se ha denominado cross-cultural comparison (Pullen, 2010: 5).

Los comienzos de las comparaciones analógicas se pueden encontrar dentro del paradigma evolucionista de la antropología y la arqueología de finales del siglo XIX y principios del siglo XX. Es cierto que este paradigma tenía un claro trasfondo racista y eurocentrista y que confiaba en un ilimitado progreso de la razón. De este modo, se buscaba ordenar a las culturas según un criterio clasificatorio tomando como el epítome del desarrollo a los países europeos (Trigger, 2007; Wylie, 2002; Yoffee, 2004). En las décadas del cincuenta y sesenta, la profesionalización de la arqueología y las teorías procesualistas buscaron eliminar la valoración moral sobre determinadas categorías sociales y les dieron un nuevo carácter a los estudios comparativos. A pesar de estos avances, muchas comparaciones se continuaron haciendo sin el rigor académico necesario y con el fin de buscar leyes generales de comportamiento llevando a descontextualizaciones que separaban a la sociedad de su producción material (Trigger, 2003, 2007: 43).
Esta situación generó una gran cantidad de críticas. Por un lado, las corrientes procesualistas más cercanas al (neo)positivismo sostuvieron que la analogía es una forma de inducción en la cual se le imponen condiciones del presente al pasado (Robles, 2017). Por otro lado, algunos representantes del postprocesualismo cuestionaban cierto determinismo clasificatorio entre las sociedades, el comportamiento humano y su entorno natural por lo que parecía subsistir una idea lineal del desarrollo similar al evolucionismo clásico. Otro conjunto de cuestionamientos se dirigía a problemas más profundos presentados por el particularismo histórico que establecía la imposibilidad de comparar culturas entre sí (Trigger, 1998: 12-13; 2007: 46).

La respuesta a estas críticas ha sido esbozada por diferentes autores desde enfoques diversos. Primero, desde un punto de vista historiográfico Yoffee y Sherratt (1993: 4) han señalado que la crítica postprocesual unifica a todos los arqueólogos procesuales por lo que se concentran en los errores de algunos investigadores para extenderlos a un conjunto heterogéneo. De hecho, para Trigger (2007 y 2008) hay demasiadas corrientes en ambos movimientos como para realizar estas generalizaciones y que lo importante es analizar las filosofías que fundamentan cada posición teórica.

Esto nos lleva al segundo tipo de respuestas: aquellas que parten de un punto de vista epistemológico y heurístico. Estos conceptos no siempre parecen estar correctamente separados, lo que lleva a confundir los momentos en que se emplea la analogía a la hora de estudiar el registro material (Burdukiewicz, 2006; Renfrew y Bahn, 2016).

Comenzando por la epistemología, las objeciones más importantes fueron por parte de los neopositivistas, quienes -según Wylie (1985: 105106) - caían en la falacia de proclamar la imposibilidad de una "analogía perfecta" ya que se basaban en una "ficción filosófica sobre la ciencia" que procede "de una visión de modelos filosóficos preconcebidos o elaborados a partir de reconstrucciones racionales de la ciencia tal como se presenta de forma popular" (Wylie, 2002: 7). Así fue como muchos representantes de esta corriente consideraban que la analogía solo servía para generar hipótesis (Gándara, 2006). Pero como resume Politis (2002), el valor de la analogía es que las partes a comparar (fuente y sujeto) no sean iguales, dado que de serlo no sería posible realizarla; su fuerza reside en la lógica detrás de esa comparación. 
De todos modos, el procesualismo y el postprocesualismo, hoy día, parecen coincidir en que lo hechos no existen en soledad, sino que siempre se analizan, implícita o explícitamente (y consciente o inconscientemente) desde una aproximación teórica (Yoffee, 2004). Esto se debe a que es epistemológicamente imposible inferir toda la información necesaria desde los materiales (Gándara, 1990; Politis, 2002; Robles, 2017; Wylie, 1985, 2002).

Desde el punto de vista heurístico es donde menos unidad se puede encontrar entre los autores. Estas divergencias son de esperarse dado que en la esfera de la interpretación es donde se pueden apreciar las diferencias entre las corrientes. La crítica postprocesual, por ejemplo, enfatiza los peligros de caer en generalizaciones esencialistas que clasifiquen a las sociedades humanas siguiendo unos pocos criterios y que se infieran comportamientos a partir de estas tipologías (Haas, 2001; Robles, 2017; Trigger, 2003; Yoffee, 2004). Otro de los problemas que se señalan, es la posibilidad de generar una interpretación que ligue unidireccionalmente la conducta humana con el contexto ecológico desechando otras variables analíticas (Trigger, 2008). Sin embargo, Trigger (2003: 22, 41) ha señalado que no toda comparación es evolucionista, ni que todo evolucionismo es racista, así como tampoco toda tendencia en el comportamiento humano tiene que ser considerada como fatalista.

Adicionalmente se ha resaltado que las analogías pueden emplearse para una gran variedad de fines. Por ejemplo: Politis -considerando el vínculo entre etnografía y arqueología- (2002: 70-71) destaca su valor a la hora de comprender la utilización de objetos, para la generación de modelos en donde los distintos materiales encuentren una explicación contextualizada y para entender y explorar otras formas de pensamiento. Por su parte, Yoffee (2004: 193-194) subraya que a través de las comparaciones se pueden generar clasificaciones útiles para iniciar análisis dinámicos de organización y, de manera similar, Trigger (2003: 14; Charlton, 1981) enfatiza su uso para contrastar teorías del cambio sociocultural de manera empírica. Parkinson (2002: 10; Peregrine, 2001) establece que la comparación intercultural es útil para presentar una perspectiva a largo plazo en donde se hace más fácil identificar y diferenciar los procesos sociales en escalas temporales más amplias que las disponibles en el trabajo de campo. Marcus y Feinman (1998: 10-12) marcan la importancia de aprovechar tradiciones analíticas más desarrolladas de un campo de estudio para aplicarlas en otro. Finalmente, Trigger (1998: 30) también agrega que sirven para incentivar la imaginación y plantear nuevas preguntas y respuestas.

Por estas razones las comparaciones interculturales no tienen por qué ser descartadas de antemano, lo que no implica que cualquier tipo de analogía sea permitida, sino que deben establecerse mecanismos de control. Con este fin, según Wylie (1985: 94-95; también Gándara, 2006: 19), hay que determinar la relevancia que tiene la analogía propuesta y señalar los respectivos principios de conexión entre las partes comparadas (Peregrine, 2001: 10). También hay que determinar qué datos se están buscando completar a través de este procedimiento y, en este complejo escenario, las teorías de rango medio (middle range theories) han intentado conciliar las complejas abstracciones filosóficas con la materialidad del registro arqueológico tendiendo un puente entre los análisis dinámicos y la materialidad estática (Peregrine, 2001; Yoffee, 2004: 183-185).

En definitiva, es necesario reconocer que en la arqueología (y en cualquier campo del conocimiento social), siempre, de un modo u otro, se están realizando comparaciones analógicas dado que los investigadores recurren a conocimientos previos para interpretar los restos materiales (Wylie, 1985). En términos estrictos cada sociedad, cada momento histórico e, incluso, cada objeto solo es idéntico a sí mismo. Para ofrecer algún tipo de interpretación que vaya más allá de la descripción (la cual tampoco estaría exenta de analogías) es necesario recurrir a algún tipo de estrategia comparativa (Politis, 2002; Yoffee, 2004); como categóricamente señala Charlton (1981: 130), la arqueología, como un todo "es analogía" (énfasis nuestro).

Esta situación metodológica nos lleva a la segunda premisa que consideramos importante para la presente investigación: contamos con una considerable tradición académica que se ha encargado de realizar estudios interculturales y, en mucho menor medida, a comparar las culturas mayas y minoicas. Estas investigaciones se concentraron en tópicos generales que son comunes a varias áreas de estudio tales como: el concepto de estado en las sociedades antiguas (Blanton, 1998; Feinman y Marcus, 1998; Flannery, 1998; Trigger, 2003; Yoffee, 2004), de las jefaturas (Earle, 1997, 2001; Hass, 2001; Parkinson, 2002), algunas cues- 
tiones de organización económica (Nakassis et al., 2011) y diversos modelos de interacción política y social (Baines y Yoffee, 1998; Blanton, 2004; Crumley, 1994, 2001; Feinman, 2001; Renfrew y Cherry, 1986).

Para el caso particular de Creta Neopalacial y el área maya los estudios comparativos son menos abundantes, pero importantes para nuestra investigación. Si bien el primer trabajo que planteó esta analogía fue el de Bintliff (1984), quien llamó la atención sobre las similitudes historiográficas que se pueden encontrar en las trayectorias académicas de estas áreas de estudio, el trabajo más influyente es la obra editada por Refrew y Cherry: Peer Polity Interaction and Socio-political Change (1986). Cherry fue quien aplicó este modelo para la isla de Creta, y, en ese mismo volumen, Freidel y Sabloff hicieron lo propio para el área mesoamericana. Sin embargo, a partir de esta obra, la trayectoria de estos campos de estudio se bifurcó: como detallaremos a lo largo de este trabajo, los académicos de la cultura maya fueron más fructíferos a la hora de explorar otras áreas de estudio para nutrirse de distintos modelos de interacción, mientras que los estudiosos del mundo egeo apenas fueron más allá del área mediterránea. De todas formas, en los últimos años se pueden encontrar más referencias a las comparaciones entre estas culturas, aunque por lo general se realizan de manera superficial.

La tercera premisa que habilita nuestro trabajo comparativo es que la analogía puede ser muy útil para aprovechar los desarrollos de un área de estudio y evaluar su aplicación en otra para suplir cierta falta de información. La arqueología maya ofrece una serie de ventajas para su estudio que están ausentes a la hora de estudiar la isla de Creta durante la Edad de Bronce; a saber, las fuentes escritas que se disponen o no están descifradas (los sistemas de escritura Jeroglífico y el Lineal A) o son temporalmente posteriores y elaborados en otros contextos históricos (como las fuentes griegas relacionadas con el ciclo mitológico cretense) $\mathrm{y}$, especialmente, la brecha temporal entre las poblaciones minoicas y las actuales es demasiado grande como para hacer un estudio etnográfico, etnohistórico y etnoarqueológico. Estas bondades, como sostiene Fash (1994: 190-191; también Sharer y Traxler, 2006), permitieron una fructífera relación entre antropología y arqueología que fue incorporada de manera recurrente en las investigaciones académicas.
Teniendo en cuenta lo anterior, consideramos que nuestra aproximación intercultural es relevante dado que hay cierta tradición historiográfica que ha comparado a ambas sociedades, al menos, superficialmente y porque las interacciones propias de las culturas antiguas tienen muchos puntos en común. Además, ya señalamos las ventajas que tiene la arqueología maya sobre la minoica en cuanto a su desarrollo teórico en relación con los enfoques antropológicos que arribaron relativamente tarde en los estudios minoicos. Ante esto, es que consideramos que esta analogía puede sernos de utilidad para encontrar nuevas respuestas ante los debates sobre el tipo de organización política en Creta durante el período Neopalacial.

\section{Modelos políticos para Creta minoica}

No es sencillo presentar la diversidad de enfoques que existen para explicar la dinámica política en el periodo Neopalacial cretense dado que se entremezclan muchas variables. Sin embargo, en esta sección detallaremos brevemente los distintos modelos de organización política que están presentes en la bibliografía desde una perspectiva crítica.

\subsection{El modelo de Evans}

Es difícil dimensionar lo profunda que ha sido la huella de Evans en los estudios minoicos, no sólo en la cuestión de la organización política, sino en cada aspecto relacionado con esta cultura. A nivel popular, turístico y académico se siguen reproduciendo algunos topoi acerca de la Edad de Bronce en Creta que fueron escritos hace más de 100 años.

Para empezar, debemos establecer que el arqueólogo inglés proponía que la isla de Creta estaba fuertemente jerarquizada; el centro de poder se encontraba en Cnosos en donde un monarca análogo a la figura mítica de Minos controlaba el resto de la isla y parte del Mediterráneo Oriental. Esta postura coincidía con varios paradigmas contemporáneos a Evans: en primer lugar, desde un punto de vista político, concordaba con la cosmovisión imperialista y etnocéntrica europea y con el nacionalismo expansionista inglés (Evans, 1921-1938; Sherratt, 2009, 2018); en segundo lugar, se basaba en los relatos míticos clásicos que habían sido revitalizados tras los descubrimientos de Troya, Micenas y Tirinto por parte de Schliemann y que 
parecían estar ligados a momentos particulares de la historia antigua (Cano, 2016); en tercer lugar, las investigaciones en Grecia continental y en Mesopotamia corroboraban la visión de estados territoriales sumamente centralizados con líderes fácilmente distinguibles y con intenciones expansionistas (Evans, 1921-1938: 1-32; Schoep, 2010).

Por esta razón, la estructura encontrada en Cnosos fue rápidamente denominada "palacio". De hecho, algunos autores destacan que este término agrupó una gran cantidad de significados y funciones siendo el hogar de un monarca, el corazón urbanístico, una metrópolis imperialista, el centro administrativo, el principal lugar de almacenamiento, el mayor productor de bienes de lujo y el templo religioso más importante (Driessen, 2002: 6). Así quedó configurada una escala jerárquica que lo tenía en la cúspide y al resto de los "palacios" que se iban descubriendo en una posición subordinada.

Este modelo tuvo como base hermenéutica las características arquitectónicas de las estructuras monumentales que se iban desenterrado; el tamaño de una estructura era directamente proporcional al poder político que ésta podía haber tenido (Evans, 1921-1938: 570-571; Vavouranakis, 2007: 265-266). Adicionalmente, se tomaban las similitudes entre los edificios como una prueba de sometimiento: cuantas más características arquitectónicas compartían, mayor había sido el grado de subordinación. Este mismo procedimiento se fue extendiendo a todos los aspectos culturales y, por lo tanto, se consideró que Cnosos había sido el centro de prácticamente todas las innovaciones tecnológicas y artísticas de la isla y que las restantes estructuras imitaban y emulaban a este centro como una manera de expresar su subordinación política, cultural e ideológica (Wiener, 1990).

La perspectiva religiosa fue muy importante para configurar y argumentar a favor de este modelo. Como mencionamos, una de las fuentes académicas de Evans estaba constituida por las ciudades Mesopotámicas que, en líneas generales, estaban conformadas por un palacio y un templo. Curiosamente, Evans no pudo desenterrar ninguna estructura que pueda ser identificada como un templo y por lo tanto creo una teoría ad hoc para poder zanjar los problemas que tenía su propuesta. Como ha detallado Schoep (2010), la solución fue establecer que el "palacio" funcionaba también como centro religioso, es decir, como "palacio-tem- plo". Y, por lo tanto, los monarcas que habitaban en esos espacios también poseían prerrogativas religiosas; en otras palabras, Minos había sido un "rey-sacerdote" (Evans, 1921-1938: 2). Esta solución fue realmente creativa dado que no había ninguna evidencia concreta para suponer que las funciones sacerdotales y las políticas estuviesen en una sola persona, así como tampoco contaba con ejemplos en otras culturas del Mediterráneo (Cano, 2020).

Finalmente, la economía de Creta también parecía haber estado centralizada en los "palacios" teniendo a Cnosos como el centro más importante. Sin embargo, no podemos atribuirle al arqueólogo inglés la autoría de esta hipótesis económica; más bien, aquí debemos resaltar los trabajos de Finley (1957) sobre la economía en la antigüedad. El historiador norteamericano fue quien introdujo las ideas de Polanyi (1944: 44-59) al mundo clásico definiendo al sistema económico como redistributivo, es decir, basado en un poder central que tenía como funciones básicas hacerse con las materias primas, almacenarlas y distribuirlas en forma de bienes y servicios (Finley, 1957: 135). Ciertamente, esta postura no contradecía la idea general de Evans de situar a los "palacios" como el centro de todos los aspectos de la vida social, sino que más bien, le brindaba un marco teórico que explicaba la acumulación de riquezas por parte de estos edificios (Cano, 2020).

\subsection{El modelo de Peer Polities Interaction}

El libro editado por Renfrew y Cherry en 1986 significó un punto de partida ineludible para comprender la relación entre ambos campos de estudio, no sólo por su propuesta intercultural, sino porque ningún investigador posterior fue ajeno a los trabajos reunidos en ese volumen. En la introducción, Renfrew utiliza a las ciudades-estados helénicas como punto de partida de su marco teórico con el fin de entender las interacciones entre unidades políticas sin usar los conceptos de dominación y subordinación (1). Para el autor, debían ser considerados otros modos de interacción como la competencia, la emulación y los intercambios (1-2; 8-9) y de esta manera se podían explicar las "homologías estructurales" entre unidades políticas que se hayan en mismo contexto ecológico (4-5).

Esta obra fue publicada en un contexto historiográfico en donde mucho de los postulados de 
Evans estaban siendo revisados. En 1984 se había publicado The Minoan Thalassocracy Myth and Reality editada por Hägg y Marinatos, Tres años después, los mismos investigadores editarían The Function of the Minoan Palaces, un libro con un espíritu similar al anterior. Esta segunda obra es más importante para nuestro trabajo dado que se presentaron serias objeciones a las ideas tradicionales de Evans respecto a los "palacios" (Nordfeld, 1987). Sin embargo, la mayoría de los académicos defendieron este paradigma por lo que el modelo jerárquico se mantuvo como la lógica principal organizativa para Creta Neopalacial (Hägg y Marinatos, 1987; Lindgren, 1987; Zois, 1987).

Ante este paradigma, Cherry proponía uno nuevo: Creta había estado dividida en diferentes entidades políticas que a lo largo del tiempo habían interactuado constantemente a través de procesos de imitación, emulación, competencia e intercambio (Anastasiadou, 2016; Cherry, 1986: 26-27). Este trabajo significó, por primera vez, la posibilidad de pensar un tipo de organización política diferente al planteado por Evans. Si bien su trabajo se centró en los "palacios" del período Protopalacial, el autor extiende este modelo al período Neopalacial y contó con la novedad de sacar a Cnosos del centro de la escena mostrando que las relaciones entre las regiones de la isla podían llegar a ser mucho más compleja que lo imaginado. Esto abrió el juego a otras interpretaciones y a estudios que tuvieron como eje de sus hipótesis el desarrollo regional y la diversidad cultural. No obstante, este modelo mantenía muchas de las lógicas jerárquicas que habían sido inauguradas por Evans. Por ejemplo, los "palacios" seguían siendo el corazón de la cultura minoica manejando la política regional y manteniendo el control económico y religioso de la sociedad. Del mismo modo, todas las manifestaciones culturales (sellos, frescos, cerámica y bienes primarios o de prestigio) encontraban su razón de ser por su vínculo con estas estructuras.

Con todo, el enfoque de Cherry fue muy fructífero porque encontró una explicación alternativa a la homogeneidad cultural en el registro arqueológico de Creta sin tener que caer en la idea de que Cnosos había controlado a los centros restantes. Además, se presentaba una manera diferente de interpretar los materiales, en especial, haciendo uso de una metodología comparativa y aplicando algunos conceptos antropológicos que son utilizados hasta el día de hoy.

\subsection{Nuevas críticas y nuevos modelos}

Si en los ochenta se comenzaron a cuestionar tímidamente algunos elementos del esquema general de Evans, en las siguientes dos décadas se refutaron muchas de sus bases argumentativas. Aunque las objeciones giraron en torno a una diversidad de temáticas, intentaremos brindar una pequeña síntesis.

El primer eje de las críticas estuvo centrado en la definición arquitectónica de los "palacios" y de todas las interpretaciones que se hacían a partir de ella. Muchos autores no encuentran ningún elemento para designar a estos edificios con este término dado que parece responder más a una tradición literaria que la evidencia arqueológica (Driessen, 2002, 2004; Hitchcock, 2000: 46 menciona las diversas alternativas propuestas; Palyvou, 2004). De hecho, con el descubrimiento de las denominadas "villas", no quedaba claro qué elementos arquitectónicos eran necesarios para separar ambas categorías (Hitchcock y Preziosi, 1997: 61). Del mismo modo, el hallazgo progresivo de nuevos "palacios" muestra que había demasiada diversidad entre estos edificios como para que haya una clasificación uniforme (Letesson, 2014). Más precisamente, se criticó que se realizaba una categorización que se basaba en comparar todas las estructuras con Cnosos, por lo cual, se incurría en el error de establecer una sistematización putativa en donde la diversidad era dejada de lado o matizada (Day y Relaki, 2002: 220).

Pero más importante aún es que a partir de dicha clasificación se establecían criterios jerárquicos unidireccionales: el tamaño era considerado como un rasgo definitorio del poder y las similitudes entre centros eran tomadas como lazos de subordinación (Vavouranakis, 2007). De este modo el "palacio" de Cnosos representaba la máxima jerarquía, seguido por los distintos "palacios" y por debajo de ellos las "villas", que eran consideradas como subunidades administrativas (Niemeier, 1997). Sin embargo, la evidencia material no es concluyente respecto a estas perspectivas dado que también puede ser interpretada como parte de un proceso de atomización de poder (Rehak y Younger, 1998).

El segundo conjunto de críticas al esquema jerárquico señala que no hay pruebas de la existencia de monarcas o gobernantes de ningún tipo que hayan habitado los "palacios". En este sentido, los estudios pictóricos han destacado la llamativa 
ausencia de representaciones que puedan identificarse como líderes específicos, sobre todo, si se tiene en cuenta de que hay figuras que claramente connotan poder en diversos registros iconográficos (Crowley, 1995: 483; Davis 1995). Adicionalmente, no parece haber existido un área residencial - (Shaw, 2015: 149-152)-y está probado que el famoso fresco "el príncipe de los lirios" fue una mala reconstrucción de Gillieron (Shaw, 2004) y, de modo similar, el "salón de trono" además de no pertenecer al período Neopalacial, fue polémicamente restaurado (Driessen, 2002; Niemeier, 1987; Papadoupoulos, 2005). Es decir, que la idea de Evans sobre la existencia de un rey Minos o de una monarquía con esa titulatura no tiene sustento arqueológico.

En tercer lugar, se realizaron cuestionamientos desde una perspectiva económica y administrativa. Los estudios realizados por Christakis han mostrado que los almacenes de estos edificios y de las "villas" no contaban con la capacidad suficiente como para llevar a cabo un sistema redistributivo centralizado. De hecho, teniendo como ejemplo a Cnosos, este edificio solamente pudo haber mantenido a 15.000 personas, muy por debajo de la cantidad que se estima que vivían en sus alrededores (Christakis 2011: 202; Keßler 2015). Por lo que no se podría haber llevado a cabo el sistema redistributivo que planteaban Polanyi, Finley y más cercano en el tiempo, Renfrew (1972) y Branigan (1988), sino que hay que postular otras dinámicas de movilización de bienes (Cano, 2018; Earle, 2011). Desde el punto de vista administrativo tampoco parece haber existido una centralización alrededor de Cnosos. Por un lado, los documentos escritos y el sistema de sellado parecen indicar que no existió un régimen centralizado de control y que tampoco, a pesar de la difusión de la escritura Lineal A, se puede sostener la existencia de un solo procedimiento administrativo, sino que más bien, cada región optó por una organización particular y que no siempre la administración estuvo centrada en los "palacios" (Schoep, 1999; Weingarten, 1989).

Sin embargo, en el aspecto religioso sí que se pueden encontrar dinámicas de interacción llamativas. Como establecimos, Evans encontró en el "palacio" de Cnosos una gran cantidad de materiales relacionados con prácticas religiosas que lo llevaron a pensar que en ese edificio habitaba una especie de rey-sacerdote que concentraba tanto el poder político como el religioso. A pesar de lo controvertido de esta postura, hay un consenso generalizado respecto a la importancia religiosa de los "palacios"; de hecho, algunos autores han aventurado la posible existencia de una teocracia administrada por una elite que basaba su poder en el control del culto (Krattenmaker, 1995; Pelon, 1995; Platon, 1983). Adicionalmente, estos edificios parecen haber iniciado un proceso de control sobre otros espacios religiosos que tradicionalmente no estaban ligados a ellos: en el período Neopalacial disminuye la cantidad de santuarios de altura respecto al período anterior al mismo tiempo que se monumentalizan siguiendo el estilo arquitectónico de los "palacios" (Rehak, 1997).

¿Cómo se explica, entonces, que no haya la suficiente evidencia para sostener una centralización política ni económica entorno los "palacios" y que al mismo tiempo estos edificios hayan concentrado una gran cantidad de actividades religiosas? Según algunos investigadores, Cnosos pudo haber sido el centro cosmológico o ideológico de Creta (Niemeier, 2004; Soles, 1995) y las elites de este lugar utilizaron su vínculo con el mundo sobrenatural como una manera de legitimar su posición social (Vansteenhuyse, 2002). Por lo tanto, el interés de estos grupos pudo haber estado más ligado a acceder a diversos vínculos con las divinidades que a un dominio político coercitivo. De manera análoga, las elites de las otras regiones pudieron haber conformado una estrategia similar a la de Cnosos utilizando el lenguaje simbólico de Cnosos para justificar su posición social (Gkiasta, 2010).

Teniendo en cuenta lo anterior, el paisaje político de Creta Neopalacial se nos presenta como un verdadero rompecabezas, el cual se hace aún más complejo si tenemos en cuenta que el período Neopalacial no fue una etapa unitaria, sino que parece haber atravesado por diferentes momentos de centralización y descentralización (Driessen y MacDonald, 1997). Por lo tanto, nos encontramos con un dinamismo que rompe el esquema tradicional de Evans y que también desafía la atomización presentada por Cherry. Ante este panorama se hizo evidente la necesidad de nuevos enfoques que permiten mayor flexibilidad en los análisis.

\subsection{Facciones y competencias}

Tanto el esquema de Evans como el de Cherry planteaban formas de interacción monolíticas en donde el centro de las manifestaciones culturales se encontraba ya sea en Cnosos o repartida entre 
los "palacios" regionales. Por esta razón, surgió la necesidad de incorporar nuevas perspectivas que pudieran explicar mejor la variabilidad del registro arqueológico desde una perspectiva dinámica. Precisamente, con este tipo de críticas comienza el trabajo de Hamilakis (2002: 179-180).

El arqueólogo griego toma como punto de partida el trabajo de Cherry dado que hace suyas las críticas que el autor le realizó al modelo tradicional de Evans (Hamilakis, 2002: 183). Sobre todo, es clave para su estudio señalar que la homogeneidad del registro material no tiene por qué ser explicada desde una lógica de dominio y subordinación, sino que probablemente se haya debido a un proceso de emulación (Hamilakis, 2002: 183). El otro elemento importante para el autor es el conflicto, por lo que sostiene que hay una gran cantidad de evidencia de violencia que atenta contra la idea pacifista que se tuvo de los minoicos durante muchos años (Hamilakis, 2002: 184).

Sin embargo, se desprende de la postura de Cherry dado que éste enfatiza demasiado el rol de los "palacios" dejando de lado las otras estructuras (Hamilakis, 2002: 185). Ante esto, Hamilakis propone introducir el concepto de facciones como una manera de comprender la competencia entre grupos que tienen la misma identidad cultural. Esta aproximación tiene la ventaja de estar sostenida desde diferentes disciplinas como la antropología, la historia y la arqueología y permite realizar comparaciones interculturales para resolver la falta de evidencia que existe en la arqueología minoica (Hamilakis, 2002: 186-187). También, considera al poder como una prerrogativa dinámica y fluctuante en donde la agencia de los sujetos y de los grupos humanos juegan un rol mayor que en los otros modelos en donde la estructura se impone sobre los individuos (Hamilakis, 2002: 187).

Para Hamilakis, los "palacios" y las otras estructuras monumentales de la isla, no serían meras imitaciones de Cnosos, sino que formarían parte de un estilo arquitectónico que tenía como razón de ser la realización de reuniones sociales y ceremoniales para reafirmar los lazos de las elites con el resto de la población tanto a nivel local como interregional (Hamilakis, 2002: 188-189, 193). Esta premisa se sostiene desde diversas perspectivas: en primer lugar, los edificios más importantes no se encuentran separados del tejido urbano, sino que lo conforman sin que haya una separación destacable; en segundo lugar, los diferentes sistemas administrativos serían indicadores de facciones organizando su respectivos territorios; y tercero, la gran cantidad de edificaciones con características "palaciales" y el consumo conspicuo de bienes de elite mostrarían la necesidad de emplear una simbología de carácter religioso para mostrarse cercanos a las divinidades (Hamilakis, 2002: 194196).

Esta hipótesis del arqueólogo griego fue realmente novedosa porque utilizó los trabajos de Brumfiel (1994) en el área azteca para explicar por qué había tantas similitudes en el registro arqueológico de las diferentes regiones sin tener que usar el paradigma de la subordinación. Además, supo darles una justificación teórica más profunda a los conceptos de competencia y emulación que Cherry mencionaba, pero no explicaba por qué las elites las habían empleado. Por otro lado, la aproximación de Hamilakis significó otro ejemplo de comparación intercultural ya que se basa en conceptos aplicados en un área de estudio ajena al investigador para resolver un problema en su propio campo.

Pero esta aproximación no se ve exenta de problemas argumentativos. Uno de los más importante corresponde al propio concepto de facciones: por un lado, al igual que Brumfiel, solo se considera una definición de facción dejando de lado otras perspectivas presentes en los estudios antropológicos (Boissevain, 1987; Bujra, 1973; Silverman y Salisbury, 1977); por otro lado, esta definición sostiene que las facciones no son grupos estables en el tiempo y que tan solo se unen por una situación particular tras la cual sus miembros vuelven a dispersarse (Day y Relaki, 2002). La gran duda que se genera es cómo estos grupos pudieron estar unidos la suficiente cantidad de tiempo para elaborar las estructuras monumentales características del período y para mantener una producción material relativamente constante durante aproximadamente 200 años. Otro de los problemas que se presenta es que tampoco delimita las arenas (el marco en el cual se inscribe el conflicto) y si sus dimensiones fueron locales, regionales o abarcaban todo el territorio insular (Day y Relaki, 2002).

\subsection{Antropología y households}

El mismo año en que Hamilakis propuso su teoría de las facciones, Driessen (2002) planteaba que en Creta había predominado una organización social corporativa basada en la solidaridad y la interdependencia de diversos subgrupos y comenzaba 
a explorar en profundidad las posibilidades analíticas de estos conceptos. Así, desde el punto de vista arquitectónico sostenía que los espacios centrales de los "palacios" eran el escenario de rituales públicos de consumo de alimentos para favorecer la cohesión social (Driessen, 2004). Dicha función hundiría sus raíces en momentos tempranos de la historia en donde se habrían construido por primera vez e indicarían cierto rasgo comunal constitutivo de la sociedad cretense (Driessen, 2004).

Ya en el año 2010 su teoría toma una forma más acabada dado que postula que los aspectos políticos y económicos de Creta podían explicarse utilizado el concepto de casa (house) y de house society ideado por Levi-Strauss y utilizado por Gillespie (2000b) para el área mesoamericana (Driessen, 2010). Para el autor (Driessen, 2010: 41):

"The attractive part in this concept is that
Houses as communities leave sufficientspa-
ce for agency, practice, structuration, and
interaction, allowing individual actors to
manipulate their place within multiple so-
cial contexts at the same time as it de-em-
phasizes certain elements of affiliation
(kin) -so difficult to identify by archaeolo-
gists- whereas stressing others (place) -so
easy to identify by archaeologists."

Así, en la práctica, este concepto tiene dos ventajas analíticas: por un lado, casi todos los modos de interacción propuestos para la isla de Creta podrían ser explicados por la flexibilidad de comportamientos que se admite dentro de esta teoría. Por otro lado, de este tipo de organización en casas (o households) se derivarían el resto de las dinámicas en materia social, política, económica y religiosa por lo que serviría para analizar la diversidad de vínculos entre grupos sociales tanto a nivel local como regional.

El centro del análisis de Driessen está puesto en la evidencia arquitectónica dado que en la perspectiva del autor ésta materializa el comportamiento de los grupos corporativos (Driessen, 2010: 41-42). Según él, la disposición de los edificios de distintos yacimientos mostraba que había unidades domésticas demasiado grandes para ser habitadas solo por una familia nuclear (contra Whitelaw, 2001) por lo que considera que debieron haber sido la sede de distintas casas (Driessen, 2010: 35). Además, el autor considera que las casas portaban una biografía que las consolidaba como espacios simbólicos de memoria colectiva ya que su historia comenzaría en el Neolítico (Driessen, 2010: 43-48). Por esta razón, el autor ve a las casas como el principal actor político y económico (Driessen, 2010: 48-53).

Finalmente, desde la perspectiva de Driessen, los conflictos entre facciones que señala Hamilakis durante el periodo Neopalacial se tratarían de una intensificación de las rivalidades entre diferentes casas, lo que explicaría el aumento del consumo de bienes de prestigio $y$, hacia el final de este período, algunas habrían prevalecido y otras nuevas habrían surgido (Driessen, 2010: 55).

En el año 2018, Driessen retoma la temática de los "palacios" para volver a criticar aquellas teorías que consideran que estos edificios revelan un tipo de poder jerárquico dado que estas posturas solamente tienen en cuenta las estrategias basadas en las redes interpersonales de los líderes (network strategies) y no consideran las estrategias corporativas (corporate strategies) (Driessen, 2018: 291-292). Ahora bien, la novedad de este trabajo está en que relaciona estas estrategias, particularmente la corporativa, con su teoría general de las household como principio constitutivo de larga duración de las poblaciones de la isla de Creta (Driessen, 2018: 294).

De este modo, considera que la acción colectiva es una de las fuerzas sociales más importantes de la historia minoica; por ejemplo, la propiedad y el trabajo comunitario de las tierras podría explicar muchas de las dinámicas económicas que no eran consideradas por el modelo redistributivo. De manera similar, propone que los "palacios" pudieron haber sido construcciones colectivas ya que su uso estaba destinado a rituales de consumo de alimento dado que sus patrones de circulación parecen haber sido fácilmente accesibles desde el exterior (Driessen, 2018: 301-303). Haciendo uso de una aproximación intercultural propone que los "palacios" podrían haber sido Great Houses en las que no solo confluirían las prácticas integrativas, sino que también podrían ser considerados espacios en donde el poder se disputaba a través de diferentes estrategias de participación y exclusión (Driessen, 2010: 306).

El último trabajo de Driessen fue publicado a principios del 2020 y forma parte de una obra colectiva para analizar el concepto de household en diferentes momentos de la historia de Creta. Está fuera de la intención de este trabajo reseñar todas las investigaciones de este volumen, pero resaltaremos la idea general que lo atraviesa. En primer 
lugar, la introducción por parte de los editores (Driessen y Relaki, 2020) resalta algunos de los puntos que mencionamos al inicio de este articulo: la falta de interés por la antropología de parte de los arqueólogos que estudian la isla de Creta y la importancia que tiene esta disciplina en los estudios del Nuevo Mundo.

Por su parte, Driessen junto a Letesson brindan una actualización teórica del término acuñado por Levi-Strauss para adaptarlo a los estudios de Creta. Según ellos, el concepto de household parte de una realidad arqueológica concreta como lo son las unidades domesticas que indican una mayor cantidad de personas que las pertenecientes a la familia nuclear (Driessen y Letesson, 2020: 7). A partir de ahí se desprenderían una serie de comportamientos comunes en donde prevalecen las interacciones comunitarias y corporativas sobre las relaciones jerárquicas. Por esta razón, los espacios destinados para realizar festines, la dificultad de encontrar relaciones jerárquicas verticales, los vínculos horizontales entre los grupos sociales y el hecho de compartir un vocabulario simbólico común serían indicadores de la preeminencia de los rasgos corporativos que constituyen a las households (Driessen y Letesson, 2020: 10-13).

Para justificar esta afirmación, los autores realizan un breve análisis diacrónico por las diferentes etapas históricas: en el período Prepalacial se habrían configurado las casas, en las cuales sus miembros habrían tenido una identidad compartida según la evidencia en las residencias, los lugares de culto y las tumbas; en el período Protopalacial, la creación de los primeros palacios serían un indicio de que ciertas households habrían logrado diferenciarse de sus pares aunque su uso parece haber estado destinado a prácticas comunitarias; finalmente, en el período Neopalacial, los "palacios" habrían estado bajo el control de algunas casas que los utilizaron para afianzar su hegemonía en rituales de participación comunal. En este contexto, la competencia entre ellas las habría llevado a comportarse como facciones (Driessen y Letesson, 2020: 13-17).

A partir de allí, las distintas contribuciones en este volumen intentan encontrar en el registro arqueológico los indicios que prueben la existencia de este tipo de dinámicas sociales. De más está decir que la mayoría de los participantes concuerdan con la postura de los editores por lo que el concepto de house society sirve para explicar una diversidad de fenómenos culturales.
Sin embargo, hay un trabajo que llama la atención sobre los problemas que tiene la utilización de esta teoría. Nanoglou manifiesta los recaudos que hay que tener al aplicar este término y a los cuales adherimos: en primer lugar, pareciera que las casas intentan resolver la tensión entre el antiguo concepto de parentesco y las formas trascendentes de organización social; en segundo lugar -y como consecuencia de lo anterior- esta teoría termina siendo tan amplia que no puede ser considerada como un modelo analítico sino que debería ser tomada como un elemento heurístico; en tercer lugar, la idea de House Society se utiliza como un modelo preconcebido al cual se intenta adaptar la evidencia material en vez de estudiar cuáles son los principios estructurales que esta evidencia sugiere (Nanoglou, 2020: 275-276). Con cierta cautela epistemológica, el autor señala que se ha pasado de considerar a las casas como una unidad de organización con cierto acervo empírico a consolidarse como un tipo de social trascendiendo los límites de su alcance como concepto. De acuerdo con su postura, hay que resolver la relación paradigmática de identificar hasta qué punto la evidencia material expresa relaciones sociales $y$, de manera análoga, como estás relaciones sociales actuaron sobre los objetos que rescata la arqueología (Nanoglou, 2020: 277-278; Relaki, 2020).

Desde nuestra óptica podemos agregar que el concepto de casa está abarcando tantos niveles de explanación que le confiere un rasgo eminentemente esencialista. Así, las households son utilizadas para explicar: la materialidad de las unidades domésticas (Knappett, 2020; Sofianou y Brogan, 2020; Gerontakou et al., 2020); la construcción de espacios públicos (Militello, Palio y Figuera, 2020); el sistema de organización social general (house society) (Drieseen y Letesson, 2020; Nanoglou, 2020; Relaki, 2020); las relaciones jerárquicas (network) y horizontales (corporate) en escalas locales y regionales de poder (Driessen y Letesson, 2020); la propiedad de la tierra y la administración de la economía (Apostolaki, 2020; Relaki, 2020); los niveles de participación en rituales religiosos (Peatfield y Morris, 2020; Puglisi, 2020); y la expresión simbólica de dicha pertenencia (Finlayson, 2020; Simandiraki-Grimshaw, 2020). En resumidas cuentas, el concepto de casa parece poder aplicarse a expresión cultural más allá de los concreta o abstracta que sea y por eso consideramos que se está cayendo en un círculo hermenéutico. 
A pesar de las críticas y de la necesidad de establecer el alcance epistemológico y hermenéutico de los conceptos de household y House Society, debemos resaltar que la flexibilidad y la apertura que ofrece esta teoría permite realizar interpretaciones más dinámicas y complejas que las que brinda el modelo clásico basado en la jerarquía y la centralidad de los "palacios". Adicionalmente, esta postura parece inaugurar una nueva relación teórico-metodológica con otras áreas de estudio y con otras disciplinas (especialmente la antropología y la etnoarqueología) favoreciendo los análisis interculturales.

\section{Debates sobre la organización política maya}

El análisis analógico que proponemos surge de que ambos campos de estudios comenzaron a emplear una metodología intercultural casi al mismo tiempo y encontraron un temprano punto de encuentro en la obra editada por Renfrew y Cherry (1986). A partir de esta publicación, los investigadores de la cultura maya profundizaron esta tendencia aventurándose a incorporar una diversidad de áreas de referencia y nuevas perspectivas teóricas mientras que los de la cultura minoica buscaron resolver los debates internos a su propio campo.

Es cierto que la arqueología maya cuenta con una serie de ventajas que han favorecido esta situación: la documentación escrita disponible y la existencia de población indígena generan que el diálogo entre la antropología, la historia y la arqueología sea más fluido permitiendo nuevas formas de interdisciplinariedad como la etnohistoria y la etnoarqueología (de Montmollin, 1989). Como resultado estos académicos han sido pioneros y más prolíferos al proponer diversos modelos de interacción y organización política. La intención de este apartado es exponer y analizar críticamente estos modelos prestando especial atención a las teorías y perspectivas que se emplean como base argumentativa para la interpretación del registro arqueológico. De este modo, podremos evaluar hasta qué punto los estudios sobre los mayas pueden ser de utilidad para la arqueología minoica.

\subsection{Las primeras síntesis}

Esta perspectiva se conformó tomando dos tipos de registros: por un lado, la evidencia arqueología producida por las elites y, complemen- tariamente, se utilizó una estereotipación de las poblaciones mayas del presente (Runggaldier y Hammond, 2016 [2007]). De este modo, quedó constituida una visión paradigmática de esta sociedad: lo que hoy consideramos ciudades mayas habrían sido centros ceremoniales donde gobernaba una casta sacerdotal. Estos sitios eran utilizados para ritos periódicos a los cuales las poblaciones que habitaban en las cercanías se acercaban para participar (Fash, 1994; Fox et al., 1996). Se asumía, por lo tanto, que el poder estaba en manos de una especie de teocracia que gobernaba sobre grupos dispersos que tenían una agricultura de subsistencia (síntesis realizada por Demarest, 2004: 43-44). De manera similar, tampoco se consideraba que los Mayas se hubiesen constituido en estados (Runggaldier y Hammond, 2016 [2007]).

Esta interpretación era coloquialmente llamada la posición de "Harvard" a la cual se le opuso otra que fue conocida como la postura de "Penn" (Universidad de Pensilvania) que abogaba por una sociedad maya más compleja y que consideraba los grandes complejos arquitectónicos como ciudades que sí habían estado pobladas. Como indican Diane y Arlen Chase (2015: 4), hacia inicios de la década del 80 el conocimiento sobre las formas de organización e interacción sociopolítica dentro de cada sitio y de los sitios entre sí era prácticamente desconocida. De acuerdo con estos autores, cada una de las interpretaciones estaba sesgada por las fuentes que habían utilizado: la corriente de "Harvard" se basaba en las fuentes etnohistóricas recogidas por los primeros conquistadores españoles y la tradición de "Penn" tomaba como eje las excavaciones arqueológicas que mostraban una organización social mucho más compleja (Chase y Chase, 2015).

El mismo tipo de debates se extendía a otras manifestaciones culturales: en el caso de la religión se dudaba entre una división entre los cultos de la elite y del resto de la población o si los ritos se concentraban alrededor de la figura del monarca y contaban con mayor participación; respecto a la economía el problema consistía en saber si la agricultura era de subsistencia o si respondía a las necesidades de un estado complejo a la vez que no estaba claro cómo funcionaba el sistema de intercambios a nivel regional; finalmente, respecto a la jerarquía de los yacimientos, se consideraba que el tamaño de los centros ceremoniales era un indicador de la importancia que había tenido determinado sitio y de ahí se especulaban qué relacio- 
nes había tenido con los restantes (Chase y Chase, 2015).

Muchas de estas perspectivas apriorísticas iban a ser lentamente abandonadas gracias a dos cambios progresivos en el mundo académico del área maya: el desciframiento de su escritura y la constante profesionalización de la arqueología. Evidentemente, ninguno de estos dos fenómenos terminó con los debates, pero sí que generaron teorías mejor fundamentadas y menos guiadas por los puntos de vista de los investigadores de turno. En definitiva, brindaban una base epistemológica para postular nuevas perspectivas, refutarlas y reformularlas partiendo de las fuentes escritas y del trabajo arqueológico (Fash [1994: 182-187] hace un interesante resumen). De este modo se configuraron los modelos que ahora pasaremos a detallar.

\subsection{Los modelos descentralizados}

En post de la brevedad agrupamos bajo este título todos aquellos modelos que parten de la base de que el estado maya tenía una centralización débil (Foias, 2013: 60; García, 2012). De hecho, este tipo de esquemas fueron los primeros que se propusieron, aunque en la mayoría de las veces se trataba de explicar la diversidad de centros urbanos extrapolando teorías de otros campos de estudio, por ejemplo, la idea de las ciudades-estado y del feudalismo. Un claro ejemplo de la primera de esta tendencia se puede encontrar en los trabajos de Schele y Freidel (1990: 39) en donde directamente se describen a las ciudades mayas como ciudades-estados similares a las poleis griegas clásicas: "pequeños países autónomos políticamente, pero interdependientes cultural, social y económicamente".

En el caso del feudalismo fue utilizado para explicar las relaciones entre los centros entendiendo que cada uno de ellos controlaba un territorio y que, dado que sus tamaños diferían, se consideraba que los más grandes se imponían sobre los pequeños (Adams y Jones, 1981: 301-302; de Montmollin, 1989: 193). Dicha propuesta se basaba en un análisis geográfico y ecológico de que combinaba la distribución de las ciudades, su cercanía y lejanía y las nuevas herramientas provenientes de la arqueología del paisaje (especialmente, polígonos de Thiessen) que permitían establecer zonas de influencia entre los yacimientos.

La verdadera novedad teórica la constituyó la participación de Freidel y Sabloff en el libro edita- do por Renfrew y Cherry (1986). Respecto a Freidel, él sostiene que nunca hubo sobre las ciudades mayas ningún tipo de nación unificada o imperio, manteniéndose una configuración de peer polities (Freidel, 1986: 93). Según su postura, los aspectos ideológicos de esta sociedad no les hubieran permitido que ninguna ciudad se imponga sobre la otra dado que la guerra era una práctica que solo involucraba a la elite y que tenía la finalidad de darle prestigio a sus miembros (Freidel, 1986: 106-108).

Por parte de Sabloff (1986), las semejanzas en la cultura maya se explicaban de una manera similar a la de la cultura minoica estudiada por Cherry: además de la competencia entre las partes, la interacción pacífica había dado como resultado un marco cultural común a todas las ciudades. Las elites son consideradas como el motor de dichos contactos y las diferencias de importancia entre los sitios en el período clásico y su posterior colapso (o "realineación") se debe a que se habría roto la interdependencia entre las ciudades haciendo caer a todo el sistema (Sabloff, 1986: 114-115).

En la década del 90 se consolidan los siguientes principios de descentralización: 1 . las ciudades eran estados que controlaban débilmente centros de segundo y tercer orden, 2 . el poder de los líderes se debía a su carisma y al prestigio que lograban a través de la guerra, rituales y las alianzas matrimoniales y 3. no había control económico del estado por lo que su legitimación ritual no era sencilla (Ball y Taschek, 1991; Foias, 2013: 60; García, 2012).

Fox, por ejemplo, es uno de los representantes de la teoría de que los Mayas se constituyeron en "estados segmentarios" (Fox et al., 1996). De acuerdo con él y sus colaboradores, la mitología y los testimonios etnohistóricos y etnográficos mostraban que la sociedad maya estaba organizada en linajes segmentarios, lo que quiere decir que las generaciones más jóvenes que veían frustradas sus aspiraciones al poder debían enlistar a sus seguidores para separarse del linaje principal o migrar a un nuevo lugar para establecerse como líderes (Fox et al.., 1996; Izquierdo y de la Cueva, 2004; Lucero, 1999; Marcus y Feinman, 1998). Esto generaba diferentes relaciones entre los grupos como la fisión, la fusión y las alianzas de modo tal que, cuando lograban imponerse como el grupo gobernante de determinada ciudad, "the state elite maintained fairly loose center-to-periphery control through ritual and kinship" (Fox et al., 1996: 818- 
819). Así, el tipo de organización social se reproduciría en la forma del estado impidiendo que se constituya los niveles jerárquicos necesarios para lograr una centralización de las ciudades a nivel regional (de Montmollin, 1989: 19-25; Fash et al., 2004; Vilella, 1993: 15-17).

Asimismo, Demarest acepta -críticamentemuchas de las propuestas del estado segmentario, pero establece que a lo largo de la historia maya pudieron haber existido otras formas de organización políticas y otras dinámicas de configuración social (Fox et al., 1996). Ante esto, adapta los enfoques antropológicos de Tambiah y de Geertz y propone el modelo de entidades galácticas (Galatic Model) que se basa en una inestabilidad endémica de las elites gobernantes mayas para mantenerse en el poder dada "the extreme dependence of leadership on ideology and ritual, and the difficulty, given these features, of sustaining cumulatively evolving states" (Fox et al., 1986: 823). El autor considera que se puede explicar los cambios en las áreas de influencia de cada entidad política, así como también la volatilidad interna que tuvieron a lo largo de su historia y su necesidad constante de utilizar los rituales para consolidar su posición social (Fox et al., 1986: 823).

Como establece Villela (1993: 17-18), en el modelo galáctico la jerarquía se conforma bajo la forma de mandala, en la cual se puede encontrar un centro con periferias de diferentes características que tienen entre sí limites fluidos y no firmemente centralizados. Otra manera de explicarlo es considerando que existían territorios multiestados en donde los líderes podían estar subordinados a otros y, a la vez, contar con seguidores de distintos rangos más allá de los lazos de parentesco (Lacadena y Ciudad, 1998; Traxler y Sharer, 2016). Esta hipótesis, al igual que la del estado segmentario, no está construida solo desde la evidencia arqueológica, sino que tiene en cuenta los testimonios transmitidos por la epigrafía e iconografía respecto a los líderes de las ciudades y su relación entre ellos (Ball y Taschek, 1991; Lacadena y Ciudad 1998).

\subsection{Las críticas al estado segmentario y la con- figuración del modelo centralizado}

Como han resaltado Diane y Arlen Chase (1996: 803) dentro de la teoría general del estado segmentario se encuentran un gran conjunto de conceptos que terminan por restarle coherencia a esta postura. Dicha diversidad hace que se presenten una gran cantidad de críticas que apuntan a diferentes aspectos. Por ejemplo, Marcus y Feinman (1998: 7-8) señalan que "ciudad-estado" es un concepto problemático porque en la actualidad existe un importante debate en los estudios clásicos sobre si las poleis griegas eran efectivamente estados (Anderson, 2009) y porque se aplica esa terminología a las ciudades mayas, las cuales no se asemejan a esta forma sociopolítica (Runggaldier y Hammond, 2016 [2007]). Del mismo modo, la utilización del término "feudal" traslada un modelo hermenéutico utilizado en un campo de estudio a otro de manera superficial.

Sin embargo, a pesar de la diversidad de teorías que incluye el modelo descentralizado, la hipótesis de los estados segmentarios parece haber sido la más representativa y, por lo tanto, la más criticada. En primer lugar, Diane y Arlen Chase (1998, 2015: 10) cuestionan que se considere que la guerra fue una acción meramente ritual como expresaba Freidel ya que las conquistas están bien documentadas arqueológica y epigráficamente. En segundo lugar, sostienen que no hay evidencia suficiente para afirmar que los linajes segmentarios sean la estructura de organización social y cuestionan que de un tipo de linaje se derive directamente una tipología de estado (Chase y Chase, 1996: 803-804; también Marcus y Feinmann, 1998: 7-8). Por esta razón, critican que este modelo atenta contra la complejidad que se puede notar en el registro arqueológico dado que se constituye como "una forma teórica idealizada" (Chase y Chase, 1996: 804). En tercer lugar, si bien aceptan que las ciudades mayas hayan sido estados (pero no "ciudades-estados") rechazan que solamente hayan tenido un carácter religioso-ritual, sino que también tenían funciones políticas y administrativas (Chase y Chase, 1996: 805). Por último, hacen una meta lectura del modelo descentralizado catalogándolo como una simplificación y una denigración de los logros de los Mayas que repite algunos prejuicios realizados por los españoles con los que entraron en contacto (Chase y Chase, 1996: 803 y 810).

Ante estos cuestionamientos, ellos proponen que la organización política maya era centralizada y fuertemente jerarquizada dado que debían controlar ciudades con una gran cantidad de personas (Chase y Chase, 1996: 805). Este control se reproducía a nivel regional y producía una jerarquía vertical que se puede analizar por medio de la gran cantidad de estamentos burocráticos 
existentes, en los patrones de asentamiento, en los testimonios epigráficos y en el acceso a ciertos recursos clave (Chase y Chase, 1996: 808-809, 2015: 10; Lucero, 1999: 212-213). Por esta razón, están a favor de que los Mayas formaron grandes estados regionales con ciudades que cumplieron la función de ser capitales (Chase y Chase, 1998 y 2004).

Aunque esta idea pareciera llevar la discusión a otro nivel, la posibilidad de que los mayas se hayan constituido como uno o varios imperios se encuentra en el origen de los debates. La idea de feudalismo buscaba establecer cierto orden jerárquico entre las ciudades a pesar de que no pudieran encontrarse lazos políticos que muestren el control de una de ella sobre las restantes. De hecho, es curioso notar como Adams, quien introdujo esta idea, es utilizado como antecedente tanto en las posturas descentralistas como en las centralistas. Esto se debe a que escribió que los Mayas habían tenido estados regionales durante el período Temprano y que en el Clásico se habían desintegrado dando lugar a una organización feudal (Lucero, 1999). Los descentralistas concuerdan con que las ciudades mayas no eran lo suficientemente fuertes para constituirse como estados y estados territoriales; los centralistas, por su parte, sostienen que algunas ciudades controlaron a otras y extendieron su dominio sobre distintas regiones también durante el período Clásico (Lucero, 1999).

Las capitales de estos estados regionales habrían sido Tikal, Calakmul y Caracol en las cuales se habrían asentado dinastías monárquicas que habrían logrado construir grandes obras de infraestructura más allá de los edificios religiosos (Foias, 2013: 60-61). De este modo, estas ciudades no solo habrían sido centros rituales (contra Sanders y Webster, 1988), sino que habrían tenido funciones administrativas y burocráticas destinadas a controlar a las restantes entidades políticas. Para lograr este objetivo, las elites habrían superado los lazos basados en el parentesco (entre ellos, el segmentario) para establecer otro tipo de vínculos que les permitiera consolidar su poder local y regionalmente generando un estamento burocrático de elites intermedias (Chase, 1994; Chase y Chase, 1994; Foias, 2013: 61; Traxler y Sharer, 2016: 49).

Tal vez por esta razón, en los últimos años, los Chase han comenzado a explorar el concepto de household como una manera de organización e interacción social que supera los límites impuestos por el parentesco (Chase y Chase, 2004). Según esta percepción, las relaciones establecidas entre las grandes ciudades respecto a las restantes permitirían la concentración del poder en manos de grupos de elite locales favorecidos por sus pares ubicados en las capitales -en este caso Caracolsin la necesidad de relaciones de parentesco entre ellos.

\subsection{Entre la descentralización y la centraliza- ción: los modelos alternativos}

Como escribe Foias (2013: 61), para la crítica postmoderna ambos modelos terminaban por configurar una visión estática de la sociedad maya que no representaba todos los cambios que habían existido a lo largo de su historia. Ante esto, una de las alternativas que se propuso fue el "modelo dinámico" desarrollado por Marcus.

Según la autora, la historia de las investigaciones sobre el pueblo maya mostraba que lo que se estaba intentando explicar era cómo podía haber existido una homogeneidad cultural a escala regional y mientras había una diversidad a nivel local (Marcus, 1993: 111-114). En cierta medida, ella consideraba que los modelos anteriores lograban expresar alguna faceta de la realidad arqueológica, pero que siempre contaban con un problema que no podían resolver, lo que les restaba valor hermenéutico (Marcus, 1993: 114-115). Marcus señala que las ciudades mayas eran formas estatales antiguas, es decir, que en ella convivían una diversidad de estrategias jerárquicas que no eran isomórficas, sino que se transformaban según el tiempo y las necesidades y que, para comprender dichos cambios, es necesario incluir las fuentes etnohistóricas para aproximarnos a la mentalidad de las poblaciones de aquella época (Braswell et al., 2004; García, 2012; Marcus, 1993;).

Marcus describe que había al menos 3 niveles de organización jerárquica: el primero era un control territorial por una especie de gobernador (halach unic); en el segundo nivel había un grupo de nobles del mismo linaje que controlaban los diferentes pueblos; en un tercer nivel se encontraban los poblados más alejados del centro en donde las interacciones estaban forjadas por alianzas endebles (Marcus, 1993: 119-120). Éstos no formarían parte de un modelo estático sino de uno dinámico que alternaría con momentos de centralización y descentralización entre una diversidad de asentamientos de diferente tamaño y jerarquía y con dis- 
tintos tipos de vínculos entre ellos (Marcus, 1993: 121, 133-135, 1998: 61-62). De esta manera, la autora considera que se resuelve la falsa dicotomía de optar entre una organización centralizada o descentralizada cuando las dinámicas que señala la etnohistoria y la arqueología parecen indicar que dependiendo del sitio y del momento histórico pudieron haber existido ambas fuerzas (Lacadena y Ciudad, 1998; Marcus, 1993: 164-170, 1998).

Como describe Regueiro Suarez (2017: 30), el funcionamiento interno de este modelo consistía en que el sitio central asumía la toma de decisiones políticas y administrativas propias del estado, realizaba las prácticas religiosas públicas y brindaba protección militar a los sitios de su área de influencia; mientras que éstos proveían bienes y mano de obra a manera de tributo.

El otro modelo propuesto es el que considera que los Mayas se organizaron en "superestados". Martin y Grübe parten de los descubrimientos epigráficos de Berlin quien encontró unos emblemas-glifo en los cuales se detallaba el nombre de las ciudades, el de sus gobernantes y los territorios que controlaban (Braswell et al., 2004: 219). A partir de allí, algunos investigadores propusieron que había existido al menos 4 imperios regionales con capital en las ciudades más importantes de cada región (Martin y Grübe, 1995: 41-42). Sin embargo, la gran cantidad de estos glifos y la diversidad de tamaño de los asentamientos hizo que estos autores sospecharan que había habido una gran intensidad de relaciones diplomáticas en la cual se ponía en juego de manera constante la hegemonía y la subordinación de las partes involucradas (Martin y Grübe, 1995: 42-44).

Los autores utilizan la competencia entre Calakmul y Tikal para ejemplificar su postura. De acuerdo con la evidencia -mayormente- epigráfica Tikal en un principio estaba bajo la subordinación (no necesariamente, control total) de Calakmul, pero después buscaría tener su propia área de influencia. En dicho conflicto, las unidades políticas menores habrían reclamado su propia hegemonía, lo que terminó por ocasionar una situación de enfrentamientos endémicos, por lo que nunca se pudieron consolidar estados regionales centralizados como el azteca a pesar de haber implementado una estrategia política similar (Martin y Grübe, 1995: 46).

Por esta razón, este modelo mantiene la idea general de que hubo grandes entidades políticas que buscaron maneras de controlar extensas regiones, pero no de manera directa al estilo de un imperio centralizado, sino imponiendo su supremacía para cobrar tributos o acceder a bienes y redes de intercambio en su propio beneficio (Foias, 2013: 79; García, 2012). La epigrafía estaría mostrando estos laxos lazos entre las ciudades mayas en donde el poder era constantemente negociado y renegociado en un contexto de extrema competencia en donde el control no era burocrático (es decir, no siempre imponiendo nuevos líderes en las ciudades bajo la órbita de Calakmul o Tikal), sino a través de un sistema de alianzas (Foias, 2013: 79; García, 2012). El verdadero poder de Tikal y Calakmul no se encontraba en el control efectivo de las otras ciudades, sino en estar en la cúspide de las relaciones jerárquicas e influyendo sobre la política de las restantes ciudades (García, 2012).

\subsection{Enseñanzas de la arqueología maya}

Esta síntesis que acabamos de presentar no está exenta de debates entre quienes defienden una $u$ otra postura. Por ejemplo, la principal crítica al modelo dinámico de Marcus es que sobredimensiona la evidencia del período Post Clásico y su importancia para el Clásico (Stuart, 1993). Marcus (1998: 63), por su parte, se defiende estableciendo que la idea de superestado es innecesariamente hiperbólica y que no cambia las bases de su enfoque. Otro de los puntos en conflicto es sobre la interpretación de las fuentes etnográficas y epigráficas: las primeras, corresponden a un período posterior en donde la organización maya había cambiado respecto al período clásico. Pero ¿cuánto había cambiado? Para los Chase, no tanto como para no ser consideradas, para Grübe y Martin, las fuentes epigráficas contemporáneas son más importantes porque dan una mirada emic más próxima. No es nuestra intención resolver estas cuestiones, pero sí nos interesa tener en cuenta qué rumbos está tomando la arqueología maya para saber si esta dirección puede ser beneficiosa para la arqueología minoica.

La primera tendencia que queremos destacar es que ha habido cierto desplazamiento sobre las temáticas del control, el dominio y la subordinación hacia las polifacéticas formas en que se presenta y se disputa el poder. Ante este cambio, la importancia de la antropología política es fundamental dado que el poder no es estable y estructural, sino que es una fuerza dinámica que se manifiesta de diferentes maneras y se materializa en una diversidad de estrategias (Yoffee, 2004: 31-34). 
En esta línea, citaremos el trabajo de Foias, quien dedica uno de sus trabajos a analizar profundamente los modelos políticos que mencionamos teniendo en cuenta una perspectiva antropológica y sin olvidar la importancia de la evidencia arqueológica. La autora establece que el poder es la capacidad de lograr hacer lo que uno (o un grupo) quiere hacer y de hacer que los otros también cumplan esa voluntad (Foias, 2013: 30). Sin embargo, para construir y consolidar cierta hegemonía es necesario tener algún control sobre recursos materiales e ideológicos para establecer relaciones desiguales de reciprocidad entre las partes (Foias, 2013: 29-32).

Según la autora, la manera que las elites tienen para establecer estos vínculos se da por medio de distintas estrategias que buscan institucionalizar, naturalizar y legitimar la desigualdad como la coerción directa o por medios menos violentos -pero no por eso menos efectivos- como la modificación del paisaje, la administración de los rituales, la vinculación con las divinidades, el acceso a determinados bienes de prestigio, el manejo de relaciones que podríamos llamar diplomáticas entre grupos similares ubicados en otros territorios y la exposición y difusión de material epigráfico e iconográfico (Foias, 2013: 32-37; Sharer, 1993; Sharer y Traxler, 2006: 82; Yoffee, 2004: 40).

En el caso de los Mayas, y contrariamente a lo planteado por el modelo centralizado, el feudal y el de los estados regionales, la importancia del poder no se encontraba en el control directo de un territorio, sino en los lazos personales establecidos entre las elites para poder movilizar recursos y personas (Foias, 2013: 171-172; Vázquez et al., 2016: 1104). Por lo que en los momentos de crisis la actividad de las elites se incrementa de tres maneras: primero, aumentan las interacciones para establecer nuevas alianzas; segundo, utilizan más parafernalia (religiosa, iconográfica y epigráfica) para legitimarse; y tercero, aumentan las interacciones con la finalidad de conseguir los materiales para producir bienes de prestigio (Arnauld et al., 2017; Laporte, 2004; Villela, 1993).

Teniendo en cuenta lo anterior, se puede comprender por qué la mayoría de los modelos fracasan en sus intentos de explicar la organización política maya: básicamente, la respuesta es que los Mayas no tenían un interés en marcar límites y fronteras, ni en establecer aparatos burocráticos centralizados, sino que más importante era mantener relaciones interpersonales con los otros grupos gobernantes (Foias, 2003: 17; Haines et al., 2016: 175). Las cuestiones de control directo, es decir, la subordinación de una ciudad a otra e, incluso, el dominio económico terminaba por ser secundarias o instrumentales con vistas a otros fines (Mcanany, 1993).

En este aspecto, Foias (2003: 18) sostiene que las conquistas militares nunca fueron bien vistas dentro de la ideología maya dado que los ancestros de los vencedores no se vinculaban con los de los vencidos y cuando una ciudad triunfaba sobre otra, no establecía un gobierno adicto, sino que incluso podía volver a poner al líder vencido para que continuara gobernando sin impedimentos. Esto no quiere decir que la guerra no haya sido un mecanismo de control o que no haya sido servido para extender la influencia de una ciudad, sino más bien, esto implica que la forma política más constante era la de la ciudad-estado independiente, más allá de que haya estado bajo la órbita de influencia de una u otra ciudad por un determinado período de tiempo (Canuto et al., 2017: 14; Foias, 2003: 19).

Esto ha llevado a que también se tengan en cuentas las ideas de Crumley (1994) respecto a las diferentes maneras de entender la jerarquía en las sociedades antiguas quien sostiene que también existen organizaciones heterárquicas en la cual diferentes elementos se clasifican siguiendo distintos parámetros. Esto no quiere decir que no existan relaciones jerárquicas, sino que una misma ciudad puede tener un rango jerárquico importante desde el aspecto religioso, pero no así desde el punto de vista político (Crumley, 1994: 5). Lo importante para el debate es considerar cómo interactuaron las fuerzas centrífugas y centrípetas de las diferentes ciudades y cómo el poder se disputó entre los grupos gobernantes (Izquierdo y de la Cueva, 2004, 2018).

La segunda tendencia que encontramos es la de relacionar al poder con las estrategias de las elites para conseguirlo, consolidarlo, expandirlo y legitimarlo. En este sentido es importante destacar el análisis de Feinman (2001) cuyo trabajo gira alrededor del cuestionamiento sobre la jerarquía y la centralización del poder, sobre todo, criticando la idea monolítica que se tiene de éste. El autor plantea dos tipos de estrategias: de exclusión (network) y las corporativas (corporate). Las primeras son propias de culturas en donde existen posiciones jerárquicas más bien vertica- 
les en donde se busca monopolizar el acceso a las fuentes de poder; en las segundas, por su parte, los accesos al poder son compartidos y los líderes tienden al anonimato dado que prevalece la importancia del grupo sobre el individuo (Foias, 2013). Lo interesante es destacar que estas estrategias no se excluyen entre sí, sino que lo importante es evaluar cuál de estas prevalece (Feinman, 2001: 156).

Para hacerlo, los investigadores han ahondado en las formas de organización social como una manera de inferir qué tipo de dinámica política predominó sobre la otra. De hecho, esta idea no es del todo nueva: precisamente, la base del modelo segmentario se constituye a partir de la hipótesis de que las relaciones sociales eran de esta misma naturaleza. De manera similar, se ha incorporado el concepto de Household o de House Society (Lévi-Strauss, 1982) para considerar un tipo de vínculo social que vaya más allá del parentesco, pero que, a la vez, lo contenga.

Sin embargo, esta idea no fue adoptada directamente de la obra del antropólogo estructuralista francés, sino que fue Gillespie (2000a) quien la aplicó a la arqueología mesoamericana. Según la autora, este concepto debe reemplazar al de linaje dado que tiene en cuenta, no sólo las relaciones sanguíneas, sino la variedad de vínculos sociales que van más allá del parentesco; y, como no está basado en modelos ideales, resulta un modelo heurístico más pragmático (Gillespie, 2000b: 468476). En lo que concierne a los debates sobre el poder y los modelos políticos en la cultura maya, si bien la autora no se decanta por ninguna de las posibilidades mencionadas, sí establece que las House Societies tienden a ser corporativas por lo que cuentan con más autonomía y pueden generar más conflictos entre las elites (Gillespie, 2000a; Regueiro, 2017).

En definitiva, hoy en día priman los análisis que entienden que el poder se manifiesta de una manera dinámica en las distintas esferas que se pueden encontrar en una cultura. Esta característica hace que sea importante analizarlo en sus procesos de construcción, legitimación, difusión y disputa y no solamente cuando ya se encuentra consolidado en determinado momento de la historia. Esto resulta un verdadero desafío ya que se trata de imprimirle dinamismo a un registro arqueológico que por sus características se presenta como estático.

\section{Conclusiones}

Cuando planteamos el análisis comparativo entre estos campos de estudio, apenas contábamos con algunas referencias imprecisas sobre la cultura maya realizadas por algunos investigadores de la cultura minoica. Dichas menciones, servían para ejemplificar la posibilidad de un ordenamiento político diferente del modelo jerárquico centrado en Cnosos postulado por Evans. Si bien coincidimos con que dicho modelo no refleja la realidad arqueológica de Creta, es cierto que la percepción sobre la organización política maya era demasiado simplista y sin el rigor necesario como para significar una alternativa teórica. Ante esto, la intención principal de este trabajo fue la de adentrarnos en este campo de estudio para explorar si la comparación y las analogías podían ser fructíferas.

Con este fin, el primer paso fue explorar la cross-cultural comparison como una metodología válida para estudiar estas dos sociedades geográfica y temporalmente alejadas. Así, hemos presentado las ideas de quienes cuestionan esta metodología y de aquellos que la defiende llegando a la conclusión que es imposible evadir la analogía como herramienta tanto epistemológica como heurística. En el caso de la arqueología maya la comparación analógica es ineludible por la importancia de los testimonios recogidos durante el dominio español y por la existencia de poblaciones mayas contemporáneas. Para los estudios minoicos, la cross-cultural comparison permite utilizar los progresos realizados por investigadores de otros campos para postular nuevas perspectivas teóricas y metodológicas.

Eso no quiere decir que en los estudios minoicos no se hayan realizado algunos esfuerzos en establecer un intercambio con otros sectores del conocimiento; de hecho, hemos señalado que el libro editado por Renfrew y Cherry contó con trabajos que analizaban ambas áreas de estudio. Sin embargo, también mencionamos que a partir de esta publicación los estudiosos de la cultura maya profundizaron esta tendencia, mientras que los arqueólogos e historiadores sobre el mundo minoico se limitaron a las referencias vagas que ya señalamos o a buscar las influencias de otras culturas del Cercano Oriente. Por esta razón, en este trabajo buscamos profundizar sobre este primer campo que consideramos que no estaba estudiado con suficiente profundidad. 
Recién en los primeros años de este siglo se comenzaron a utilizar enfoques con cierto acercamiento a la antropología, como las facciones postuladas por Hamilakis y los primeros trabajos de Driessen que lo llevaron a postular, de forma más concisa en 2020, una organización social basada en households. Curiosamente, muchas de las teorías y ejemplos utilizados en estos trabajos fueron tomadas de los académicos del área maya, lo que volvió a generar un acercamiento entre estos campos y evidenció la necesidad de volver a los trabajos analógicos comparativos con las enmiendas necesarias para evadir los problemas que generaron estas posturas en el pasado.

En lo que compete a las finalidades de este trabajo destinado a los modelos de organización e interacción política, los académicos que analizan la arqueología maya han presentado una gran cantidad de posibilidades para entender las dinámicas políticas durante el período Clásico. Esta proliferación de propuestas no solo se debe a la relación cercana entre la arqueología y la antropología, sino que también hay una generosa cantidad de fuentes escritas disponibles que permiten un tipo de interpretación que en la arqueología minoica no se puede realizar. Obviamente no es solo una cuestión de cantidad de modelos, sino también de los aspectos cualitativos que presentan y de las herramientas teóricas y metodológicas que se utilizan en los debates que han generado. En este sentido, consideramos que los estudios minoicos se pueden ver realmente favorecidos: al estudiar los aportes epistemológicos y hermenéuticos empleados para el área maya, se pueden postular diferentes alternativas a las formas de organización política posibles para la sociedad minoica del período Neopalacial.

De hecho, los análisis sobre las relaciones políticas en el área maya permiten hacer ciertos paralelismos con los estudios de la cultura minoica. En primer lugar, el modelo centralizado se asemeja en sus fundamentos al modelo de Evans en cuanto que defiende la existencia jerarquías fuertes y verticales con estados controlando grandes extensiones de territorio y con una organización burocrática centrada en la capital; la gran diferencia, sin embargo, es que los Chase admiten la presencia de al menos 3 capitales en constante competencia entre sí, mientras que Evans solo reconoce a Cnosos en ese rol.

En segundo lugar, la idea del estado segmentario maya se basa en muchos de los fundamentos esta- blecidos por el modelo de Peer Polities Interaction por lo que las similitudes con la propuesta de Cherry son evidentes. Sin embargo, en el primer caso, el valor de las relaciones de parentesco es muy importante para la configuración de ese esquema, situación que no se da en el caso minoico dado que no se cuenta con esta información. Otra diferencia, es que Cherry también postulaba estados fuertes y jerárquicos para Creta y era tan solo a nivel regional en donde disputaba la hegemonía; en cambio los modelos descentralizados, en general, sostienen que las elites mayas tuvieron serios problemas en imponerse sobre otras ciudades y que incluso debían ser muy cautos en el tipo de relaciones que establecían con sus subordinados a nivel local.

Ante esto, el modelo galáctico postuló que ante dicha inestabilidad los límites y el control entre las unidades políticas eran realmente fluidos y que la naturaleza del poder entre los Mayas era tan débil que dependía de los rituales religiosos para difundir la ideología dominante que funcionara como nexo cohesionador social. Paradójicamente, esta idea también ha sido propuesta para la Edad de Bronce en Creta, ipero para defender totalmente lo contrario!: Soles (1995) -basándose superficialmente en el caso de Teotihuacán- postuló que Cnosos había dominado toda la isla, pero que la base de dicho poder era de carácter ideológico y religioso y no (únicamente) político. Es decir, una centralización con bases menos coercitivas. Lo curioso es que es innegable la importancia ritual que tuvieron las estructuras monumentales minoicas y su relación con otros espacios de culto, pero sin embargo no se ha postulado ningún modelo que plantee que la proliferación de la parafernalia religiosa pueda ser una característica de debilidad en las relaciones jerárquicas en distintos niveles sociales o políticos.

Así como Hamilakis y Driessen utilizaron los trabajos académicos del área maya para elaborar sus propias aproximaciones teóricas, es interesante plantear la posibilidad de emplear algunos de los postulados del modelo galáctico para explorar nuevas respuestas ante los tradicionales debates. De hecho, se podría complementar esta hipótesis con algunas propuestas de Marcus y su modelo dinámico por lo que habría que considerar los momentos de centralización y descentralización existentes en Creta durante el período Neopalacial y la posibilidad de que no haya existido solo una manera de vincularse entre los cen- 
tros, sino que las relaciones de poder pudieron haber variado según las entidades políticas que entraban en contacto.

De manera similar, habría que considerar si en algún momento Cnosos, Festos y Malia no pudieron haber constituido sus áreas de influencia sin haber tenido que controlar directamente esos territorios; es decir, al estilo de los superestados propuestos por Martin y Grübe. Es cierto que esta última propuesta está largamente basada en la evidencia epigráfica, pero eso no quita que algunos principios de organización no se hayan manifestado de manera similar o que otros centros de menor jerarquía hayan estado bajo una u otra zona de influencia o incluso haber contado con cierta autonomía. Esperamos continuar con este camino de análisis en futuros trabajos.

Para finalizar, es clave destacar que en la actualidad ambos campos de estudio han cambiado el punto de vista sobre cómo analizar los modelos políticos: ya no se estudia al poder como una atribución propia de los grupos de elite o de los estados, sino que se considera que éste se produce dinámicamente a través de las relaciones sociales y por medio de procesos de adquisición, construcción, legitimación, competencia y resistencia de las hegemonías en diversas arenas de disputa. Por esta razón, la incorporación a este debate de las estrategias network y corporate como herramientas analíticas sirve para señalar que las relaciones de poder son más complejas de lo que se pensaba anteriormente.

Asimismo, se ha profundizado sobre los vínculos que existe entre las relaciones sociales y las dinámicas políticas por medio del concepto de household y de house society. De igual modo, desde nuestra postura, se corre el riesgo de ligar una tipología social con un tipo de ordenamiento político de manera directa y casi fatalista. Por esta razón, mencionamos que los estudios sobre las household parecen tener un rasgo esencialista en cuanto que todos los fenómenos culturales (incluyendo, sobre todo, los materiales) parecen ser una manifestación de este tipo de ordenamiento social.

Así y todo, estas nuevas aproximaciones abren el abanico de posibilidades a maneras más complejas de examinar las relaciones de poder y sus dinámicas a través del tiempo y de las diferentes culturas y permiten generar nuevas formas de conocimientos que nutren nuestra comprensión sobre las sociedades antiguas.

\section{Agradecimientos}

Quisiera agradecer a Paola Ramundo y a Pilar Regueiro Suárez por su ayuda bibliográfica respecto a la cultura maya y a los evaluadores de que permitieron aumentar su calidad argumentativa. Pero sobre todo quisiera agradecer a Maria Anastasiadou a Diana Wolf y a todo el equipo de CMS de la Universidad de Heidelberg por inspirar este trabajo a partir de los debates que se generaron durante mi estadía en dicha institución.

\section{Bibliografía}

ADAMS, Ellen. 2004: "Power and Ritual in Neopalatial Crete: a Regional Comparison". Word Archaeology, 36 (1), pp. 26-42.

ADAMS, Ellen. 2006: "Social Strategies and Spatial Dynamics in Neopalatial Crete: An Analysis of the North-Central Area". American Journal of Archaeology, 110 (1), pp. 1-36.

ADAMS, Richard; JONES, Richard. 1981: "Spatial Patterns and Regional Growth among Classic Maya". American Antiquity, 46 (2), pp. 301322.

ANASTASIADOU, Maria. 2016: "Drawing the Line: Seals, Script, and Regionalism in Protopalatial Crete". American Journal of Archaeology, 120 (2), pp. 159-193.

ANDERSON, Greg. 2009: "The Personality of Greek State". Journal of Hellenic Studies, 129, pp. 1-22.

APOSTOLAKI, Emmanouela. 2020: "Whom the House Concerned. Land Ownership and Lines of Descent in Prehistoric Crete". En M. RELAKI y J. DRIESSEN (eds.): Oikos. Archaeological approaches to House Societies in the Bronze Age Aegean, pp. 97-120. Presses universitaires de Louvain. Lovaina.

ARNAULD, Marie; ANDRIEU, Chloé; FORNE, Mélanie. 2017: "'In the days of my life.' Elite Activity and Interactions in the Maya Lowlands from Classic to Early Postclassic Times (the Long Ninth Century, AD 760-920)". Journal de la Société des américanistes, Maya Times, pp. 41-96.

BALL, Joseph; TASCHEK, Jennifer. 1991: “Late Classic Lowland Maya Political Organization and Central-Place Analysis: New Insights from the Upper Belize Valley". Ancient Mesoamerica, 2, pp. 149-165.

BAINES, John; YOFFEE, Norman. 1998: “Order, 
Legitimacy, and Wealth in Ancient Egypt and Mesopotamia". En G. FEINMAN y J. MARCUS (eds.): Archaic States, pp. 199-260. School of American Research Press. Santa Fe.

BINTLIFF, John. 1984: "Structuralism and Myth in Minoan Studies". Antiquity, 58 (222), pp. 3338.

BLANTON, Robert. 1998: "Beyond Centralization. Steps Toward a Theory of Egalitarian Behavior in Archaic States". En G. FEINMAN y J. MARCUS (eds.): Archaic States, pp. 135-172. School of American Research Press. Santa Fe.

BLANTON, Robert. 2004: "Settlement Pattern and Population Change in Mesoamerican and Mediterranean Civilizations: A Comparative Perspective". En S. ALCOCK y J. CHERRY (eds.): Side-by-Side Survey: Comparative Regional Studies, pp. 206-242. Oxbow Books. Oxford.

BOISSEVAIN, Jeremy. 1987: "Factions". En J. KUPER (ed.): Political Science and Political Theory. Routledge Library Editions. Political Thought and Political Philosophy. Routledge \& Kegan Paul Books Ltd. Londres y Nueva York.

BOURDIEU, Pierre; CHAMBOREDON, Jean-Claude; PASSERON, Jean-Claude. 2004: El oficio del sociólogo. Presupuestos epistemológicos. Siglo XXI. Buenos Aires.

BRANIGAN, Keith. 1981: "Minoan Colonialism". Annual of the British School at Athens, 76, pp. 23-33.

BRANIGAN, Keith. 1988: "Social Security and the State in Middle Bronze Age Crete". Aegaeum 2, pp. 11-16. Lovaina. Peeters Publishers.

BRASWELL, Geoffrey; PRAGER, Christian; BILL, Cassandra; SCHWAKE, Sonja; BRASWELL, Jennifer. 2004: "The Rise of Secondary States in the Southeastern Periphery of the Maya World. A Report on Recent Archaeological and Epigraphic Research at Pusilha, Belize". Ancient Mesoamerica, 15, pp. 219-233.

BRUMFIEL, Elizabeth. 1994: "Factional Competition and Political Development in the New World: An Introduction". En E. BRUMFIEL y J. FOX (eds.): Factional Competition and Political Development in the New World, pp. 3-14. Cambridge University Press. Cambridge.

BUCK, Robert. 1962: "The Minoan Thalassocracy re-examined". Historia: Zeitschrift fur Alte Geschichte, 11, pp. 129-137.

BUJRA, Janet. 1973: "The Dynamics of Political Action: A New Look at Factionalism". American Anthropologist, New Series, 75 (1), pp. 132-152.
BURDUKIEWICZ, Jan. 2006: "Epistemological Status of Archaeology - Current Controversies". Archeologia Polona, 44, pp. 73-88.

CALDESI VALERI, Valerio. 2009: Minos of Cnossos: King, Tyrant and Thalassocrat. Tesis Doctoral. University of Texas Austin.

CANO MORENO, Jorge. 2016: "Política, economía y religión en Creta Neopalacial. Hacia la construcción de una identidad de elite". En R. FLAMMINI y J.M. TEBES (eds.): Interrelaciones e identidades culturales en el Cercano Oriente Antiguo, pp. 201-238. IMHICIHU-CONICET. Argentina.

CANO MORENO, Jorge. 2018: "Cuestionando el concepto de redistribución en Creta Neopalacial: un aporte desde los sistemas de sellado". Sociedades Precapitalistas, 8 (1).

CANO MORENO, Jorge. 2020: "Una introducción al problema de la organización política en Creta durante el período Neopalacial". Revista de Estudios Clásicos, 49, pp. 45-101.

CANUTO, Marcello; BARRIENTOS, Tomás; LAMOUREUX-ST-HILAIRE, Maxime; BUSTAMANTE, Eduardo. 2017: "La Casa de los Tronos: El Palacio de La Corona y el gobierno hegemónico de Kaanal”. En B. ARROYO; L. MÉNDEZ SALINAS y L. PAIZ (eds.): XXX Simposio de Investigaciones Arqueológicas en Guatemala 2016, pp. 185-198. Ministerio de Cultura y Deportes. Instituto de Antropología e Historia. Asociación Tikal. Guatemala.

CHARLTON, Thomas. 1981: "Archaeology, Ethnohistory, and Ethnology: Interpretive Interfaces". Advances in Archaeological Method and Theory, 4, pp. 129-176.

CHASE, Arlen. 1994: "Elites and the Changing Organization of Classic Maya Society". En D. CHASE y A. CHASE (eds.): Mesoamerican Elites. An Archaeological Assessment, pp. 30-49. University of Oklahoma Press. Norman.

CHASE, Arlen; CHASE, Diane. 1994: "Mesoamerican Elites: Assumptions, Definitions, and Models". En D. CHASE y A. CHASE (eds.): Mesoamerican Elites. An Archaeological Assessment, pp. 3-17. University of Oklahoma Press. Norman.

CHASE, Arlen; CHASE, Diane. 1996: "More Than Kin and King: Centralized Political Organization among the Late Classic Maya". Current Anthropology, 37 (5), pp. 803-810.

CHASE, Arlen; CHASE, Diane. 1998: "Late Classic Maya Political Structure, Polity Size, and War- 
fare Arenas". En A. CIUDAD; M. FERNÁNDEZ; J. GARCÍA; M. IGLESIAS; A. LACADENA y L. SANZ (eds.): Anatomía de una civilización: aproximaciones interdisciplinarias a la cultura maya, pp. 11-29. Sociedad Española de Estudios Mayas. Madrid.

CHASE, Daiane; CHASE, Arlen. 2004: "Archaeological Perspectives on Classic Maya Social Organization from Caracol, Belize". Ancient Mesoamerica, 15, pp. 139-147.

CHASE, Daiane; CHASE, Arlen 2015: "Thirty Years of Archaeology at Caracol, Belize: Retrospective and Prospective". Research Reports in Belizean Archaeology, 12, pp. 3-14.

CHERRY, John. 1986: "Polities and Palaces: Some Problems in Minoan State Formation". En C. RENFREW y J. CHERRY (eds.): Peer Polity Interaction and Socio-Political Change, pp. 19-46. Cambridge University Press. Cambridge.

CHRISTAKIS, Kostis. 2011: "Redistribution and Political Economies in Bronze Age Crete". Forum. Redistribution in Aegean Palatial Societies, American Journal of Archaeology, 115, pp. 197-205.

CROWLEY, Janice. 1995: "Images of Power in the Bronze Age Aegean". En R. LAFFINEUR y W.D. NIEMEIER. (eds.): Politeia. Society and State in the Aegean Bronze Age, pp. 476-491, Vol. 2. Université de Liège. Lieja.

CRUMLEY, Caroline. 1994: "Heterarchy and the Analysis of Complex Societies". Archeological Papers of the American Anthropological Association, 6 (1), pp. 1-5.

CRUMLEY, Caroline. 2001: "Communication, Holism, and the Evolution of Sociopolitical Complexity". En J. HASS, (ed.): From Leaders to Rulers, pp. 19-36. Springer Science+Business Media. New York.

DAVIS, Ellen. 1995: Art and Politics in the Aegean: The Missing Ruler. En P. REHAK (ed.): The Role of the Ruler in the Prehistoric Aegean, pp. 1119. Université de Liège. Lieja.

DAVIS, Jack. 2008: "Minoan Crete and the Aegean Islands". En C. SHELMERDINE (ed.): The Cambridge Companion to the Aegean Bronze Age, pp. 186-209. University Press. Cambridge.

DAY, Peter; RELAKI, Maria. 2002: "Past Factions and Present Fictions: Palaces in the Study of Minoan Crete". En J. DRIESSEN; I. SCHOEP y R. LAFFINEUR (eds.): Monuments of Minos. Rethinking the Minoan Palaces, pp. 217-234. Université de Liège. Lieja.
DEMAREST, Arthur. 2004: Ancient Maya: The Rise and Fall of a Rainforest Civilization. Cambridge University Press. Cambridge.

DE MONTMOLLIN, Oliver. 1989: The Archaeology of Political Structure. Cambridge University Press. Cambridge.

D0W, Sterling. 1967: "The Minoan Thalassocracy". Proceedings of the Massachussets Historical Society, 7, pp. 3-32.

DRIESSEN, Jan. 2002: “The King Must Die.' Some Observations on the use of Minoan Court Compounds". En J. DRIESSEN; I. SCHOEP y R. LAFFINEUR (eds.): Monuments of Minos. Rethinking the Minoan Palaces, pp. 1-14. Université de Liège; Austin: University of Texas at Austin, Program in Aegean Scripts and Prehistory. Liege.

DRIESSEN, Jan. 2004: "The Central Court of the Palace at Knossos". En G. CADOGAN; E. HATZAKI y A. VASILAKIS (eds): Knossos: Palace, City, State, pp. 75-82. British School of Athens Studies 12. The British School at Athens. Nottingham.

DRIESSEN, Jan. 2010: "Spirit of Place: Minoan Houses as Major Actors". En D. PULLEN (ed.): Political economies of the Aegean Bronze Age, pp. 35-65. Oxford and Oakville. Oxbow Books.

DRIESSEN, Jan. 2018: "Beyond the Collective ... The Minoan Palace in Action". En M. RELAKI y Y. PAPADATOS (eds.): From the Foundations to the Legacy of Minoan Archaeology, pp. 291313. Oxbow Books. Oxford y Filadelfia.

DRIESSEN, Jan; LETESSON, Quentin. 2020: “On the House'. A Diachronic Look on the Configuration of Minoan Social Relationships". En M. RELAKI y J. DRIESSEN (eds.): Oikos. Archaeological approaches to House Societies in the Bronze Age Aegean, pp. 7-24. Presses universitaires de Louvain. Lovaina.

DRIESSEN, Jan; RELAKI, Maria. 2020: "House Rules..." En M. RELAKI y J. DRIESSEN (eds.): Oikos. Archaeological approaches to House Societies in the Bronze Age Aegean, pp. 1-6. Presses universitaires de Louvain. Lovaina.

DRIESSEN, Jan; MACDONALD, Colin. 1997: The Troubled Island: Minoan Crete Before and After the Santorini Eruption. Université de Liège, Histoire de l'Art et Archéologie de la Grèce Antique. Lieja.

EARLE, Timothy. 1997: How Chiefs Come to Power. Stanford University Press. Stanford.

EARLE, Timothy. 2001: "Institutionalization of 
Chiefdoms: Why Landscapes are Built". En J. HASS (ed.): From Leaders to Rulers, pp. 105124. Springer Science+Business Media. Nueva York.

EARLE, Timothy. 2011: "Redistribution and the Political Economy: The Evolution of an Idea". Redistribution in Aegean Palatial Societies. American Journal of Archaeology, 115, pp. 237-244.

EVANS, Arthur. 1921-1938: The Palace of Minos at Knossos: a comparative account of the successive stages of the early Cretan civilization as illustred by the discoveries at Knossos. Macmillan. Londres.

FASH, William. 1994: "Changing Perspectives on Maya Civilization". Annual Review of Anthropology, 23, pp. 181-208.

FASH, William; ANDREWS, Wyllys; MANAHAM, Kam. 2004: "Political Decentralization, Dynastic Collapse, and the Early Postclassic in the Urban Center of Copan, Honduras". En A. DEMAREST; P. RICE y D. RICE (eds.): The Terminal Classic in the Maya Lowlands, pp. 260287. University Press of Colorado. Colorado.

FEINMAN, Gary. 2001: "Mesoamerican Political Complexity. The Corporate-Network Dimension". En J. HASS (ed.): From Leaders to Rulers, pp. 151-176. Springer Science+Business Media. New York.

FLANNERY, Kent. 1998: "The Ground Plans of Archaic States". En G. FEINMAN, G. y J. MARCUS (eds.): Archaic States, pp. 15-58. School of American Research Press. Santa Fe.

FINLAYSON, Sarah. 2020: "The House Seal. Examining Seal Use and Sealing Practices in Proto- and Neopalatial Crete in the Light of Lévi- Strauss' Model of House Societies". En M. RELAKI y J. DRIESSEN (eds.): Oikos. Archaeological Approaches to House Societies in the Bronze Age Aegean, pp. 185-200. Presses universitaires de Louvain. Lovaina.

FINLEY, Moses. 1957: "The Mycenaean Tablets and Economic History". Review of Economic History, 10, pp. 128-141.

FOX, John; COOK, Garrett; CHASE, Arlen; CHASE, Diane. 1996: "Questions of Political and Economic Integration: Segmentary Versus Centralized States among the Ancient Maya". Current Anthropology, 37 (5), pp. 795-801.

FOX, John; COOK, Garrett; DAMAREST, Arthur. 1996: "Constructing Maya Communities: Ethnography for Archaeology". Current An- thropology, 37 (5), pp. 811-830.

FOIAS, Antonia. 2003: "Perspectivas teóricas en las dinámicas del estado Clásico maya: resultados preliminares del Proyecto Eco-arqueológico Motul de San José, 1998-2003". Mayab, 16 , pp. 15-32.

FOIAS, Antonia. 2013: Ancient Maya Political Dynamics. Gainesville. University Press of Florida.

FREIDEL, D. 1986: "Maya Warfare: An Example of Peer Polity Interaction". En C. RENFREW y J. CHERRY (eds.): Peer Polity Interaction and Socio-Political Change, pp. 93-108. Cambridge University Press. Cambridge.

GÁNDARA, Manuel. 1990: "La analogía etnográfica como heurística”. En Y. SUGIURA y M. SERRA (eds.): Etnoarqueología. Coloquio Bosch-Gimpera, pp. 43-82. Universidad Nacional Autónoma de México. México.

GÁNDARA, Manuel. 2006: "La inferencia por analogía: más allá de la analogía etnográfica". En Consejo Superior de Investigaciones Científicas, CSIC (eds.): Etnoarqueología de la Prehistoria: más allá de la analogía, pp. 13-24. CSIC. Madrid.

GARCÍA CAPISTRÁN, Hugo. 2012: "El reino de la serpiente bajo la mirada de sus aliados". En J. ŹRAŁKA; W. KOSZKUL y B. GOLIŃSKA (eds.): Maya Political Relations and Strategies. Proceedings of the 14th European Maya Conference, pp. 41-58. Cracovia. Polish Academy of Arts and Sciences.

GERONTAKOU, Eleni; KYRITSI, Maria; SALICHOU, Alexandra. 2020: “T $\alpha \varepsilon v$ oók $\omega$. Tracing Social Identity and Structure in the Minoan town of Zakros". En M. RELAKI y J. DRIESSEN (eds.): Oikos. Archaeological Approaches to House Societies in the Bronze Age Aegean, pp. 157172. Presses universitaires de Louvain. Lovaina.

GILLESPIE, Susan. 2000a: "Lévi-Strauss: Maison and Société à Maisons". En R. JOYCE y S. GILLESPIE (eds.): Beyond Kinship. Social and Material Reproduction in House Society, pp. 22-52. University of Pennsylvania. Filadelfia.

GILLESPIE, Susan. 2000b: "Rethinking Ancient Maya Social Organization: Replacing "Lineage" with "House"'. American Anthropologist, 102, (3) pp. 467-484.

GKIASTA, Maria. 2010: "Social Identities, Materiality and Connectivity in Early Bronze Age Crete". En P. van DOMMELEN y B. KNAPP (eds.): Material Connections in the Ancient Medite- 
rranean: Mobility, Materiality and Identity, pp. 85-105. Routledge. Oxford.

HÄGG, Robin; MARINATOS, Nannó. (eds.): 1984: The Minoan Thalassocracy. Myth and Reality. Paul Åström Forlag. Estocolmo.

HÄGG, Robin; MARINATOS, Nannó. 1987: “Preface”. En R. HÄGG y N. MARINATOS (eds.): The Function of the Minoan Palaces, pp. 9-11. Paul Åström Forlag. Estocolmo.

HÄGG, Robin; MARINATOS, Nannó (eds.): 1987: The Function of the Minoan Palaces. Paul Åström Forlag. Estocolmo.

HAINES, Helen; GRAHAM, Elizabeth; SAGEBIEL, Kerry; HOWIE, Linda. 2016: "“'There is no Death! What Seems so is Transition": Difficulties in Identifying Political Boundaries between Lamanai and Ka'kabish". Research Reports in Belizean Archaeology, 13, pp. 169-179.

HAMILAKIS, Yannis. 2002: "Too many chiefs? Factional competition in Neopalatial Crete". En J. DRIESSEN; I. SCHOEP y R. LAFFINEUR (eds.): Monuments of Minos. Rethinking the Minoan Palaces, pp. 179-199. Université de Liège; Austin. University of Texas at Austin, Program in Aegean Scripts and Prehistory. Lieja.

HASS, Jonathan. 2001: "Cultural Evolution and Political Centralization". En J. HASS (ed.): From Leaders to Rulers, pp. 3-18. Springer Science Business Media. Nueva York.

HITCHCOCK, Louise. 2000: Minoan Architecture. A Contextual Analysis. Paul Aströms Förlag. Estocolmo.

HITCHCOCK, Louise; PREZIOSI, Donald. 1997: "The Knossos Unexplored Mansion and the 'Villa-Anex Complex.'” En R. HÄGG, (ed.): The Function of the "Minoan Villa". Proceedings of the Eighth International Sympsosium at the Swedish Institute at Athens, 6-8, 1992, pp. 5162. Paul Aströms Förlag. Estocolmo.

IZQUIERDO Y DE LA CUEVA, Ana. 2004: "Unidad y fragmentación del poder entre los Mayas". Estudios de Cultura Maya, XXV, pp. 57-76.

IZQUIERDO Y DE LA CUEVA, Ana. 2018: "Heterarquía y unidades corporativas. Instituciones del gobierno interno maya”. Estudios de Cultura Maya, LI, pp. 11-42.

KANTOR, Helene. 1947: "The Aegean and the Orient in the Second Millennium B. C". American Journal of Archaeology, 51 (1), pp. 1-15+17-103.

KARADIMAS, Nektarios; MOMIGLIANO, Nicoletta. 2004: “On the Term 'Minoan' before Evan's
Work in Crete". Studi Micenei ed Egeo Anatolici, 46 (2), pp. 243-258.

KEßLER, T. 2015: "A Royal Gift? Bulk Grain Storage in Protopalatial and Neopalatial Crete". Studi Micenei ed Egeo-Anatolici, NS 2015, pp. 137170.

KNAPPETT, Carl. 2020: "Architectural Facilities and House Society in Bronze Age Crete". En M. RELAKI y J. DRIESSEN (eds.): Oikos. Archaeological approaches to House Societies in the Bronze Age Aegean, pp. 81-96. Presses universitaires de Louvain. Lovaina.

KNAPPETT, Carl; NIKOLAKOPOULOU, Irene. 2008: "Colonialism without Colonies? A Bronze Age case of study from Akrotiri, Thera". Hesperia: The Journal of American School of Classical Studies at Athens, 77, pp. 1-42.

KRATTENMAKER, Kathleen. 1995: "Palace, Peak and Sceptre: The Iconography of Legitimacy". En P. REHAK (ed.): The Role of the Ruler in the Prehistoric Aegean, pp. 49-58. Université de Liège. Lieja.

LACADENA GARCÍA-GALLO, Alfonso; CIUDAD RUIZ, Andrés (1998): "Reflexiones sobre la estructura política maya clásica". En A. CIUDAD; Y. FERNÁNDEZ, J. GARCÍA; M. IGLESIAS; A. LACADENA y L. SANZ (eds.): Anatomía de una civilización: aproximaciones interdisciplinarias a la cultura maya, pp. 31-64. Sociedad Española de Estudios Mayas. Madrid.

LAPORTE, Juan. 2004: "Terminal Classic Settlement and Polity in the Mopan Valley, Peten, Guatemala". En A. DEMAREST; P. RICE y D. RICE (eds.): The Terminal Classic in the Maya Lowlands, pp. 195-230. University Press of Colorado. Colorado.

LÉVI-STRAUSS, Claude. 1982: The Way of the Masks. University of Washington Press. Washington.

LETESSON, Quentin. 2014: "From Building to Architecture: The Rise of the Configurational Thinking in Bronze Age Crete". En E. PALIOU; U. LIEBERWIRTH y S. POLLA (eds.): Spatial Analysis and Social Spaces, pp. 49-90. De Gruyter. Gottingen.

LINDGREN, Margareta. 1987: "The Function of the Minoan Palaces. Myth and Reality". En R. HÄGG y N. MARINATOS (eds.): The Function of the Minoan Palaces, pp. 39-42. Paul Åström Forlag. Estocolmo.

LUCERO, Lisa. 1999: "Classic Lowland Maya Political Organization: A Review”. Journal of World 
Prehistory, 13 (2), pp. 211-263.

MARCUS, Joyce. 1993: "Ancient Maya Political Organization". en J. SABLOFF y J. HENDERSON (eds.): Lowland Maya Civilization in the Eighth Century A.D., pp. 111-184. Dumbarton Oaks. Washington D.C.

MARCUS, Joyce. 1998: "The Peaks and Valleys of Ancient States: An Extension of the Dynamic Model". En G. FEINMAN y J. MARCUS (eds.): Archaic States, pp. 3-14. School of American Research Press. Santa Fe.

MARCUS, Joyce; FEINMAN, Gary. 1998: "Introduction". En G. FEINMAN; J. MARCUS (eds.): Archaic States, pp. 59-94. School of American Research Press. Santa Fe.

MARTIN, Simon; GRUBE, Nikolai. 1995: "Maya Superstates". Archaeology, 48 (6), pp. 41-46.

MCANANY, Patricia. 1993: "The Economics of Social Power and Wealth among Eighth-Century Maya Households". En J. SABLOFF y J. HENDERSON (eds.): Lowland Maya Civilization in the Eighth Century A.D., pp. 65-90. Dumbarton Oaks. Washington, D.C.

MELAS, Manolis. 1991: "Acculturation and Social Mobility in the Minoan World". En R. LAFFINEUR y L. BASCH (eds.): Thalassa. L'Egée préhistorique et la mer, pp. 169-188. Université de Liège. Histoire d l'art et archéologie de la Grèce antique. Lieja.

MILITELLO, Petro; PALIO, Orazio; FIGUERA, Marianna. 2020: "Houses, Central Buildings and Embedded Production Interpreting the Role of Households in Phaistos and Hagia Triada during the Palatial Periods". En M. RELAKI y J. DRIESSEN (eds.): Oikos. Archaeological approaches to House Societies in the Bronze Age Aegean, pp. 120-140. Presses universitaires de Louvain. Lovaina.

NAKASSIS, Dimitri; PARKINSON, William; GALATY, Michael. 2011: "Redistributive Economies from a Theoretical and Cross-Cultural Perspective". Forum. Redistribution in Aegean Palatial Societies, American Journal of Archaeology, 115, pp. 174-188.

NANOGLOU, Stratos. 2020: "Housing People in Aegean Prehistory". En M. RELAKI y J. DRIESSEN (eds.): Oikos. Archaeological approaches to House Societies in the Bronze Age Aegean, pp. 275-288. Presses universitaires de Louvain. Lovaina.

NIEMEIER, Wolf-Dietrich. 1987: "On the Function of the 'Throne Room' in the Palace at Knossos".
En R. HÄGG y N. MARINATOS (eds.): The Function of the Minoan Palaces, pp. 153-168. Paul Åström Forlag. Estocolmo.

NIEMEIER, Wolf-Dietrich. 1991: "Minoan Artisans Travelling Overseas: The Alalakh Frescoes and the Painted Plaster Floor at Tel Kabri (Western Galilee)". En R. LAFFINEUR y L. BASCH (eds.): Thalassa. L'Egée préhistorique et la mer, pp. 189-207. Université de Liège. Histoire $d$ l'art et archéologie de la Grèce antique. Lieja.

NIEMEIER, Wolf-Dietrich. 1997: "The Origins of the Minoan 'Villa' System". En R. HÄGG (ed.): The Function of the "Minoan Villa", pp. 15-19. Svenska Institutet i Athen Series in $4^{\circ}$, XL. Paul Åström Forlag. Estocolmo.

NIEMEIER, Wolf-Dietrich. 2004: "When Minos Ruled the Waves: Knossian Power Overseas". En G. CADOGAN; E. HATZAKI y A. VASILAKIS (eds): Knossos: Palace, City, State, pp. 393-398. British School of Athens Studies 12. The British School at Athens. Nottingham.

NORDFELD, A. 1987: "Residential Quarters and Lustral Basins”. En R. HÄGG y N. MARINATOS (eds.): The Function of the Minoan Palaces, pp. 187-194. Estocolmo. Paul Åström Forlag.

PALYVOU, Clairy. 2004: "Outdoor Space in Minoan Architecture: "Community and Privacy"'. En G. CADOGAN; E. HATZAKI y A. VASILAKIS (eds): Knossos: Palace, City, State, pp. 207-218. British School of Athens Studies 12. The British School at Athens. Nottingham.

PAPADOPOULOS, John. 2005: "Inventing the Minoans: Archaeology, Modernity and the Quest for European Identity". Journal of Mediterranean Archaeology, 18 (1), pp. 87-149.

PARKINSON, William. 2002: "Introduction: Archaeology and Tribal Societies". En W. PARKINSON (ed.): The Archaeology of Tribal Societies, pp. 1-12. International Monographs in Prehistory. Michigan.

PEATFILED, Alan; MORRIS, Christine. 2020: "Peak Sanctuary Figurines Materialising Issues of Ritual Personhood within Community/House Identity". En M. RELAKI y J. DRIESSEN (eds.): Oikos. Archaeological approaches to House Societies in the Bronze Age Aegean, pp. 173184. Presses universitaires de Louvain. Lovaina.

PELON, Oliver. 1995: "Royauté et iconographie royale dans la Créte minoenne". En R. LAFFINEUR y W.-D. NIEMEIER (eds.): Politeia. Society and State in the Aegean Bronze Age, pp. 
309-322. University of Heidelberg. Archäologisches Institut,

PEREGRINE, Peter. 2001: "Cross-Cultural Comparative Approaches in Archaeology". Annual Review of Anthropology, 30, pp. 1-18.

PLATON, Nicolas. 1983: "The Minoan Palaces: Centres of Organisation of a Theocratic, Social and Political System". En O. KRZYSZKOWSKA y L. NIXON (eds.): Minoan Society, pp. 273-280. Bristol Classical Press. Bristol.

POLANYI, Karl. 1944: The Great Transformation. Beacon Press. Boston.

POLITIS, Gustavo. 2002: "Acerca de la Etnoarqueología en América del Sur". Horizontes Antropológicos, 8 (18), pp. 61-91.

PUGLISI, Dario. 2020: "Rites of Passage in Minoan Palatial Crete and their Role in Structuring a House Society". En M. RELAKI y J. DRIESSEN (eds.): Oikos. Archaeological approaches to House Societies in the Bronze Age Aegean, pp. 63-80. Presses universitaires de Louvain. Lovaina.

PULLEN, Daniel. 2010: "Introduction: Political Economies of the Aegean Bronze Age". En D. PULLEN (ed.): Political economies of the Aegean Bronze Age, pp. 1-11. Oxbow Books. Oxford and Oakville.

REGUEIRO SUAREZ, Pilar. 2017: Las danzas de Yaxuun B'ahlam IC de Yaxchilán. Un caso de estrategia y negociación política en la cuenca media de Usumacinta durante el siglo VIII d.C. Tesis de Maestría. Universidad Nacional Autónoma de México.

REHAK, Paul. 1997: "The Role of the Religion Painting in the Function of the Minoan Villa: The Case of Ayia Triadha". En R. HÄGG (ed.): The Function of the "Minoan Villa", pp. 163-175. Paul Åström Forlag. Estocolmo.

REHAK, Paul; YOUNGER, John. 1998: "Review of Aegean Prehistory VII: Neopalatial, Final Palatial and Postpalatial Crete". American Journal of Archaeology, 102 (1), pp. 91-173.

RELAKI, Maria. 2020: "Ecologies of Care. Examining the Practices of Belonging in Bronze Age Crete". En M. RELAKI y J. DRIESSEN (eds.): Oikos. Archaeological approaches to House Societies in the Bronze Age Aegean, pp. 317-340. Presses universitaires de Louvain. Lovaina.

RENFREW, Colin. 1986: "Peer Polity Interaction and Socio-Political Change". En C. RENFREW y J. CHERRY (eds.): Peer Polity Interaction and Socio-Political Change, pp. 1-18. Cambridge
University Press. Cambridge.

RENFREW, Colin. 1972: The Emergence of Civilization. The Cyclades and the Aegean in the third millennium BC. Methuen. Londres.

RENFREW, Colin; CHERRY, John. (eds.) 1986: Peer Polity Interaction and Socio-Political Change. Cambridge University Press. Cambridge.

RENFREW, Colin; BAHN, Paul. 2016: Archaeology. Theories, Methods and Practice. Thames \& Hudson. Nueva York.

ROBLES ZAMORA, Alfredo. 2017: "El responsabilismo epistémico en la analogía etnoarqueológica: aproximaciones a una teoría de la justificación para la práctica arqueológica en México". Revista Colombiana de Filosofía de la Ciencia, 34, pp. 117-136.

RUNGGALDIER, Astrid; HAMMOND, Norman. 2016 [2007]: "Maya States: The Theoretical Background in Historical Overview". En L. TRAXLER y R. SHARER (eds.): The Origin of Mayan States, pp. 33-58. University of Pennsylvania Press. Filadelfia.

SABLOFF, Jeremy. 1986: "Interaction among Classic Maya Polities: A Preliminary Examination". En C. RENFREW y J. CHERRY (eds.): Peer Polity Interaction and Socio-Political Change, pp. 109-116. Cambridge University Press. Cambridge.

SANDERS, William; WEBSTER, David. 1988: "The Mesoamerican Urban Tradition". American Anthropologist, 90 (3), pp. 521-546.

SCHELE, Linda; FREIDEL, David. 1990: Una selva de reyes. La asombrosa historia de los antiguos mayas. Fondo de Cultura Económica. México.

SCHOEP, Ilse. 1999: "Tablets and Territories? Reconstructing Late Minoan IB Political Geography through Undeciphered Documents". American Journal of Archaeology, 103 (2), pp. 201-221.

SCHOEP, Ilse. 2010: "The Minoan "Palace-Temple" Reconsidered: A Critical Assessment of the Spatial Concentration of Political, Religious and Economic Power in Bronze Age Crete". Journal of Mediterranean Archaeology, 23 (2), pp. 219-243.

SCHOEP, Ilse. 2018: "Building the Labyrinth: Arthur Evans and the Construction of Minoan Civilization". American Journal of Archaeology, 122 (1), pp. 5-32.

SHARER, Robert. 1993: "The Social Organization of the Late Classic Maya: Problems of Definition and Approaches". En J. SABLOFF y J. HEN- 
DERSON (eds.): Lowland Maya Civilization in the Eighth Century A.D., pp. 91-110. Dumbarton Oaks. Washington, D.C.

SHARER, Robert; TRAXLER, Loa. 2006: The Ancient Maya. Stanford University Press. Stanford.

SHAW, Joseph. 2015: Elite Minoan Architecture. Its Development at Knossos, Phaistos, and Malia. INSTAP Academic Press. Filadelfia.

SHAW, Maria. 2004: "The "Priest-King" Fresco from Knossos: Man, Woman, Priest, King, or Someone Else?" Hesperia Supplements, 33, XAPIL: Essays in Honor of Sara A. Immerwahr, pp. 65-84.

SHERRATT, Susan. 2009: "Representations of Knossos and Minoan Crete in the British, American and Continental Press, 1900-c. 1930". Creta Antica, 10 (2), pp. 619-649.

SILVERMAN, Marilyn; SALISBURY, Richard. 1977: "An Introduction: Factions and the Dialectic". En M. SILVERMAN y R. SALISBURY (eds.): $A$ House Divided? Anthropological Studies of Factionalism, pp. 1-11. Memorial University of Newfoundland. Institute of Social and Economical Research.

SIMANDIRAKI-GRIMSHAW, Anna. 2020: "The Body and the Ring. Metal Rings and Social Structures in the Late Bronze Age Aegean". En M. RELAKI y J. DRIESSEN (eds.): Oikos. Archaeological approaches to House Societies in the Bronze Age Aegean, pp. 201-224. Presses universitaires de Louvain. Lovaina.

SOFIANOU, Chrysa. y BROGAN, Tom, 2020: "Darkness at the Edge of Town? Possible Signs of Inequality in the LM IB Oikos at Papadiokampos". En M. RELAKI y J. DRIESSEN (eds.): Oikos. Archaeological approaches to House Societies in the Bronze Age Aegean, pp. 140-156. Presses universitaires de Louvain. Lovaina.

SOLES, Jeffrey. 1995: "The Functions of a Cosmological Center: Knossos in Palatial Crete". En R. LAFFINEUR y W.-D. NIEMEIER (eds.): Politeia. Society and State in the Aegean Bronze Age, pp. 405-414. University of Heidelberg. Archäologisches Institut. Heidelberg.

STARR, Chester. 1955: "The Myth of the Minoan Thalassocracy". Historia: Zeitschrift für Alte Geschichte, 3, pp. 282-291.

STUART, David. 1993: "Historical Inscription and the Maya Collapse". En J. SABLOFF y J. HENDERSON (eds.): Lowland Maya Civilization in the Eighth Century A.D., pp. 321-354. Dumbarton Oaks. Washington. D.C.
TRAXLER, Loa; SHARER, Robert (eds.) 2016: The Origin of Maya States. University of Pennsylvania Museum of Archaeology and Anthropology. Fildadelfia.

TRIGGER, Bruce. 1998: "Archaeology and Epistemology: Dialoguing across the Darwinian Chasm". American Journal of Archaeology, 102 (1), pp. 11-34.

TRIGGER, Bruce. 2003: Understanding Early Civilizations. A Comparative Study. Cambridge University Press. Cambridge.

TRIGGER, Bruce. 2007: "Cross-Cultural Comparison and Archaeological Theory". En N. MESKELL; R. PREUCEL (eds.): A Companion to Social Archaeology, pp. 43-65. Malden. Blackwell Publishing Ltd.

VANSTEENHUYSE, Klaas. 2002: "Minoan Courts and Ritual Competition". J. DRIESSEN; I. SCHOEP y R. LAFFINEUR (eds.): Monuments of Minos. Rethinking the Minoan Palaces, pp. 235-245. Université de Liege. Lieja.

VÁZQUEZ LÓPEZ, Verónica; KUPPRAT, Félix; MORALES AGUILAR, Carlos; GARCÍA CAPISTRÁN, Hugo; VALENCIA RIVERA, Rogelio; 2016: "Los cambios de poder en el juego interdinástico: los gobernantes de Calakmul de los siglos V a VIII d.C.". En B. ARROYO; L. MÉNDEZ y G. AJÚ (eds.): XXIX Simposio de investigaciones arqueológicas en Guatemala 2015, pp. 11011114. Ministerio de Cultura y Deportes. Instituto de Antropología e Historia. Asociación Tikal. Guatemala.

VAVOURANAKIS, Giorgios. 2007: "Palatial Style Architecture and Power in Bronze Age Crete". En S. ANTONIADOU y A. PACE (eds.): Mediterranean Crossroads, pp. 263-289. Pierides Foundation. Atenas.

VILLELA, Khristaan. 1993: The Classic Maya Secondary Tier: Power and Prestige at Three Polities. Tesis de Maestría. University of Texas Austin.

WACE, Alan; BLEGEN Carl. 1939: "Pottery as Evidence for Trade and Colonisation in the Aegean Bronze Age". Klio Beiträge zur Alten Geschichte, 32 (32), pp. 131-147.

WEINGARTEN, Judith. 1989: "New and Old Elements in the Seals and Sealings of the Temple Repository, Knossos". En R. LAFFINEUR (ed.): Transition. Le Monde égéen du Bronze moyen au Bronze récent, pp. 39-52. Université de Liège. Lieja.

WHITELAW, Todd. 2001: "From Sites to Communities: Defining the Human Dimensions of 
Minoan Urbanism". En K. BRANIGAN (ed.): Urbanism in the Aegean Bronze Age, pp. 15-37. Sheffield Academic Press. Londres.

WIENER, Malcolm. 1990: "The Isles of Crete? The Minoan Thalassocracy Revisited". En D. HARDY (ed.): Thera and the Aegean World III, pp. 128-161. The Theran Foundation. Londres.

WIENER, Malcolm. 2015: "Oh, No- Not Another Chronology!". The Art and Culture of Ancient Egypt: Studies in Honor of Dorothea Arnold. Bulletin of the Egyptological Seminar, 19, pp. 649-663.

WYLIE, Alison. 1985: "The Reaction against Analogy". Advances in Archaeological Method and Theory, 8, pp. 63-111.

WYLIE, Alison. 2002: Thinking from Things. Essays in the Philosophy of Archaeology. University of California Press. California.
YOFFEE, Norman. 2004: Myths of the Archaic State. Evolutions of the Earliest Cities, States, and Civilizations. Cambridge University Press. Nueva York.

YOFFEE, Norman; SHERRATT, Andrew. 1993: Archaeological Theory: Who sets the Agenda? Cambridge University Press. Cambridge.

ZOIS, Antonis. 1987: "The Function of the Minoan Palace: A Contribution to the Definition of the Main Problem or a Model for Future Research". En R. HÄGG y N. MARINATOS (eds.): The Function of the Minoan Palaces, pp. 43-44. Paul Åström Forlag. Estocolmo. 